\title{
Phylogenomic analysis of 589 metagenome-assembled genomes encompassing all major prokaryotic lineages from the gut of higher termites
}

\author{
Vincent Hervé ${ }^{\text {Corresp., } 1}{ }$, Pengfei Liu ${ }^{1}$, Carsten Dietrich ${ }^{1}$, David Sillam-Dussès ${ }^{2}$, Petr Stiblik ${ }^{3}$, Jan Šobotník ${ }^{3}$, Andreas \\ Brune ${ }^{\text {Corresp. } 1}$ \\ ${ }^{1}$ Research Group Insect Gut Microbiology and Symbiosis, Max Planck Institute for Terrestrial Microbiology, Marburg, Germany \\ 2 Laboratory of Experimental and Comparative Ethology EA 4443, Université Paris 13, Villetaneuse, France \\ 3 Faculty of Forestry and Wood Sciences, Czech University of Life Sciences, Prague, Czech Republic \\ Corresponding Authors: Vincent Hervé, Andreas Brune \\ Email address: vincent.herve8@gmail.com, brune@mpi-marburg.mpg.de
}

"Higher" termites have been able to colonize all tropical and subtropical regions because of their ability to digest lignocellulose with the aid of their prokaryotic gut microbiota. Over the last decade, numerous studies based on 16S rRNA gene amplicon libraries have largely described both the taxonomy and structure of the prokaryotic communities associated with termite guts. Host diet and microenvironmental conditions have emerged as the main factors structuring the microbial assemblages in the different gut compartments.

Additionally, these molecular inventories have revealed the existence of termite-specific clusters that indicate coevolutionary processes in numerous prokaryotic lineages. However, for lack of representative isolates, the functional role of most lineages remains unclear. We reconstructed 589 metagenome-assembled genomes (MAGs) from the different gut compartments of eight higher termite species that encompass 17 prokaryotic phyla. By iteratively building genome trees for each clade, we significantly improved the initial automated assignment, frequently up to the genus level. We recovered MAGs from most of the termite-specific clusters in the radiation of, e.g., Planctomycetes, Fibrobacteres, Bacteroidetes, Euryarchaeota, Bathyarchaeota, Spirochaetes, Saccharibacteria, and Firmicutes, which to date contained only few or no representative genomes. Moreover, the MAGs included abundant members of the termite gut microbiota. This dataset represents the largest genomic resource for arthropod-associated microorganisms available to date and contributes substantially to populating the tree of life. More importantly, it provides a backbone for studying the metabolic potential of the termite gut microbiota, including the key members involved in carbon and nitrogen biogeochemical cycles, and important clues that may help cultivating representatives of these understudied clades. 


\section{Phylogenomic analysis of 589 metagenome-assembled} 2 genomes encompassing all major prokaryotic lineages from 3 the gut of higher termites

5 Vincent Hervé ${ }^{*}$, Pengfei Liu ${ }^{1}$, Carsten Dietrich ${ }^{1}$, David Sillam-Dussès ${ }^{2}$, Petr Stiblik ${ }^{3}$, Jan

6 Šobotník $^{3}$, Andreas Brune ${ }^{1 *}$

$8 \quad{ }^{1}$ Research Group Insect Gut Microbiology and Symbiosis, Max Planck Institute for Terrestrial 9 Microbiology, 35043 Marburg, Germany

$10{ }^{2}$ Laboratory of Experimental and Comparative Ethology EA 4443, Université Paris 13, Sorbonne 11 Paris Cité, Villetaneuse 93430, France

$12{ }^{3}$ Faculty of Forestry and Wood Sciences, Czech University of Life Sciences, Prague 6, Suchdol 13 16500, Czech Republic

$15 *$ Corresponding authors:

16 Vincent Hervé ${ }^{1}$

17 Research Group Insect Gut Microbiology and Symbiosis, Max Planck Institute for Terrestrial 18 Microbiology, Karl-von-Frisch-Strasse 10, 35043 Marburg, Germany

19 Email address: vincent.herve8@gmail.com

Andreas Brune ${ }^{1}$

22 Research Group Insect Gut Microbiology and Symbiosis, Max Planck Institute for Terrestrial 23 Microbiology, Karl-von-Frisch-Strasse 10, 35043 Marburg, Germany

24 Email address: brune@mpi-marburg.mpg.de

Key words: metagenome-assembled genomes, gut microbiology, higher termites

ORCID:

29 Vincent Hervé: http://orcid.org/0000-0002-3495-561X

30 Andreas Brune: http://orcid.org/0000-0002-2667-4391 


\section{Abstract}

32 "Higher" termites have been able to colonize all tropical and subtropical regions because of their ability to digest lignocellulose with the aid of their prokaryotic gut microbiota. Over the last decade, numerous studies based on 16S rRNA gene amplicon libraries have largely described both the taxonomy and structure of the prokaryotic communities associated with termite guts. Host diet and microenvironmental conditions have emerged as the main factors structuring the microbial assemblages in the different gut compartments. Additionally, these molecular inventories have revealed the existence of termite-specific clusters that indicate coevolutionary processes in numerous prokaryotic lineages. However, for lack of representative isolates, the functional role of most lineages remains unclear. We reconstructed 589 metagenome-assembled genomes (MAGs) from the different gut compartments of eight higher termite species that encompass 17 prokaryotic phyla. By iteratively building genome trees for each clade, we significantly improved the initial automated assignment, frequently up to the genus level. We recovered MAGs from most of the termite-specific clusters in the radiation of, e.g., Planctomycetes, Fibrobacteres, Bacteroidetes, Euryarchaeota, Bathyarchaeota, Spirochaetes, Saccharibacteria, and Firmicutes, which to date contained only few or no representative genomes. Moreover, the MAGs included abundant members of the termite gut microbiota. This dataset represents the largest genomic resource for arthropod-associated microorganisms available to date and contributes substantially to populating the tree of life. More importantly, it provides a backbone for studying the metabolic potential of the termite gut microbiota, including the key members involved in carbon and nitrogen biogeochemical cycles, and important clues that may help cultivating representatives of these understudied clades.

\section{Introduction}

Termites (Blattodea: Termitoidae) are eusocial insects that have predominantly and successfully colonized tropical and subtropical areas across the world. One of the keys to this success is their rare ability to degrade lignocellulose, a very abundant but recalcitrant complex carbon substrate (Cragg et al., 2015). As major decomposers, termites play an important role in carbon cycling (Yamada et al., 2005; Dahlsjö et al., 2014; Liu et al., 2015; Griffiths et al., 2019). Lignocellulose digestion by termites is attributed to the presence of a specific microbiota colonizing the different gut compartments of the host (Brune, 2014). Even though termites produce endogenous cellulases in the labial glands and/or midgut (Tokuda et al., 2004; Fujita, Miura \& Matsumoto, 2008), the digestive processes in the hindgut are the result of microbial activities.

"Lower" termites feed almost exclusively on wood, whereas "higher" termites

65 (Termitidae family) diversified their diet and extended it from wood to plant litter, humus, and soil (Donovan, Eggleton \& Bignell, 2001). Higher termites represent the most diverse and taxon- 
68

69

70

71

72

73

74

75

76

77

78

79

80

81

82

83

84

85

86

87

88

89

90

91

92

93

94

95

96

97

98

99

100

101

102

103

104

105

morphology is more complex than that of the basal clades, and is characterized by the presence of a mixed-segment and an enlarged proctodeal segment P1. Moreover, the gut displays strong variations in $\mathrm{pH}$ and oxygen partial pressure along the anterior-posterior axis, which creates microenvironments within the gut (Brune, 2014).

Termites harbor a specific and complex gut microbiota (Brune \& Dietrich, 2015; Bourguignon et al., 2018). Over the last decade, numerous studies targeting the 16S rRNA gene have cataloged the prokaryotic diversity of the termite gut microbiota. By analyzing the structure and composition of these microbial communities, the roles of host taxonomy (Dietrich, Köhler \& Brune, 2014; Abdul Rahman et al., 2015), host diet (Mikaelyan et al., 2015a), and microenvironments found in the different gut compartments (Mikaelyan, Meuser \& Brune, 2017) have emerged as the main factors shaping the termite gut microbiota. These studies have also highlighted patterns of dominant taxa associated with specific diet and/or gut compartment (Mikaelyan, Meuser \& Brune, 2017). For instance, Spirochaetes tend to be the dominant phylum in the gut of wood/grass feeders, whereas their abundance is lower in litter, humus and soil feeders, in which Firmicutes are much more abundant. The accumulated 16S rRNA gene reads have revealed the existence of termite-specific clusters among both bacterial and archaeal phyla (e.g. among Fibrobacteres, Clostridia, Spirochaetes, and Euryarchaeota).

All these studies focusing on the $16 \mathrm{~S}$ rRNA gene have helped microbiologists in answering the question "who is there?", but the following questions "what are they doing?" and "who is doing what?" remain open. Attempts to answer the latter questions have been made, e.g., by analyzing different fractions of the gut content of Nasutitermes spp., which led to the identification of fiber-associated cellulolytic bacterial taxa (Mikaelyan et al., 2014), or by focusing on the diversity of individual functional marker genes, such as nifH (Ohkuma, Noda \& Kudo, 1999) or formyl-tetrahydrofolate synthetase (Ottesen \& Leadbetter, 2011). The latter approach, however, is problematic because the organismal origin of the respective genes is often obfuscated by frequent horizontal gene transfers between prokaryotes. Thus, it has been suggested that genome-centric instead of gene-centric approaches are much more relevant for elucidation of soil or gut microbiotas (Prosser, 2015). Unfortunately, the number of available isolates of termite gut microbiota and their genomes (Zheng \& Brune, 2015; Yuki et al., 2018) are low compared to those from other environments. However, modern culture-independent methods, namely metagenomics and single-cell genomics have recently allowed the generation of numerous metagenome-assembled genomes (MAGs) and single-amplified genomes (SAGs), respectively, from uncultivated or difficult to cultivate organisms (Albertsen et al., 2013; Woyke, Doud \& Schulz, 2017). MAGs are becoming increasingly more prominent in the literature (Bowers et al., 2017) and populate the tree of life (Parks et al., 2017). Additionally, MAGs offer the opportunity to explore the metabolic potential of these organisms and to link it with their ecology.

To date, only a limited number of MAGs and SAGs of uncultured bacteria have been 
106 recovered from the guts of higher termites; these represent termite-specific lineages of 107 Fibrobacteres (Abdul Rahman et al., 2016) and Cyanobacteria (Utami et al., 2018). Here, we 108 applied a binning algorithm to 30 metagenomes from different gut compartments of eight higher 109 termite species encompassing different feeding groups to massively recover hundreds of 110 prokaryotic MAGs from these samples. After quality filtering, all these MAGs were 111 taxonomically identified within a phylogenomic framework and are discussed in the context of 112 insect gut microbiology and symbiosis.

114 Materials and Methods

\section{Metagenomic datasets}

116 To cover a wide range of microbial diversity, we used 30 metagenomic datasets representing the main gut compartments (crop, midgut, P1-P5 proctodeal compartments of the hindgut) and main

118 feeding groups present in higher-termites (see Table 1). Eight species of higher termites,

119 identified by both morphological criteria and analysis of the mitogenome, were considered:

120 Cornitermes sp., Cubitermes ugandensis, Microcerotermes parvus, Nasutitermes corniger, 121 Neocapritermes taracua, Termes hospes (Dietrich \& Brune, 2016), Labiotermes labralis and

122 Embiratermes neotenicus (Hervé \& Brune, 2017). Field experiments were approved by the 123 French Ministry for the Ecological and Solidarity Transition (UID: ABSCH-CNA-FR-240495-2; 124 permit TREL1902817S/118). Processing of the termite samples and DNA extraction and 125 purification were described previously (Rossmassler et al., 2015). Metagenomic libraries were 126 prepared, sequenced, quality controlled, and assembled at the Joint Genome Institute (Walnut 127 Creek, CA, USA). DNA was sequenced using Illumina HiSeq 2000 or Illumina HiSeq 2500 128 (Illumina Inc., San Diego, CA). Quality-controlled reads were assembled and uploaded to the 129 Integrated Microbial Genomes (IMG/M ER) database (Markowitz et al., 2014). Accession 130 numbers and information about these 30 metagenomes can be found in Table S1. Genome reconstruction

133 For each metagenomic dataset, both quality-controlled (QC) and assembled (contigs) reads were 134 downloaded from IMG/M ER in August 2017. To obtain coverage profile of contigs from each 135 metagenomic assembly, the QC reads were mapped to contigs using BWA v0.7.15 with the bwa136 mem algorithm (Li \& Durbin, 2009). This generated SAM files that were subsequently converted 137 into BAM files using SAMtools v1.3 (Li et al., 2009). Combining coverage profile and 138 tetranucleotide frequency information, genomes were reconstructed from each metagenome with 139 MetaBAT version 2.10.2 with default parameters (Kang et al., 2019). Quality of the 140 reconstructed genomes was estimated with CheckM v1.0.8 (Parks et al., 2015). Only MAGs that 141 were at least $50 \%$ complete and with less than $10 \%$ contamination, were retained for subsequent 
142 analyses. These MAGs have been deposited at the Sequence Read Archive (SRA) under the

143 BioProject accession number PRJNA560329; genomes are available with accession numbers 144 SRR9983610-SRR9984198 (Table S2). Additionally, the MAGs have been deposited at the

145 NCBI's Assembly Database under the accessions WQRH00000000-WRNX00000000 (Table 146 S2).

For each MAG, CheckM was also used to extract 16S rRNA gene sequences as well as a set of 43 phylogenetically informative marker genes consisting primarily of 29 ribosomal proteins (PF00466, PF03946, PF00298, PF00572, PF00238, PF00252, PF00861, PF00687, PF00237, PF00276, PF00831, PF00297, PF00573, PF00281, PF00673, PF00411, PF00164, PF00312, PF00366, PF00203, PF00318, PF00189, PF03719, PF00333, PF00177, PF00410, PF00380, PF03947, PF00181), 9 RNA polymerase domains (PF04563, PF04997, PF00623, PF05000, PF04561, PF04565, PF00562, PF04560, PF01192), 2 tRNA ligases (TIGR00344 and TIGR00422), a signal peptide binding domain (PF02978), a translation-initiation factor 2 (PF11987) and a TruB family pseudouridylate synthase (PF01509). Finally, CheckM was also used for a preliminary taxonomic classification of the MAGs by phylogenetic placement of the MAGs into the CheckM reference genome tree.

\section{Phylogenomic analysis}

In order to improve the initial CheckM classification, genome trees were built for each clade of interest (from kingdom to family level). Using this initial CheckM classification and when available, the 16S rRNA gene classification, genomes of closely related organisms and relevant outgroups were manually selected and downloaded from NCBI and IMG/M ER. These genomes were subjected to a similar CheckM analysis to extract a set of 43 single-copy marker genes, to translate them into amino acid sequences, and to create a concatenated fasta file $(6,988$ positions). For each clade of interest, the amino acid sequences from the MAGs, their relatives, and outgroups were aligned with MAFFT v7.305b and the FFT-NS-2 method (Katoh \& Standley, 2013), and the resulting alignment was filtered using trimAl v1.2rev59 with the gappyout method (Capella-Gutierrez et al., 2009). Smart Model Selection (Lefort, Longueville \& Gascuel, 2017) was used to determine the best model of amino acid evolution of the filtered alignment based on Akaike Information Criterion. Subsequently, a maximum-likelihood phylogenetic tree was built with PhyML 3.0 (Guindon et al., 2010). Branch supports were calculated using a Chi2-based parametric approximate likelihood-ratio test (aLRT) (Anisimova $\&$ Gascuel, 2006). Finally, each tree was visualized and edited with iTOL (Letunic \& Bork, 2019). Following the procedure described above, a genome tree containing only the MAGs

176 generated in the present study was also built and visualized with GraPhlAn version 0.9.7 177 (Asnicar et al., 2015). 

phylogenetic framework of the current SILVA reference database (SSURef NR 99 release 132)

182 (Quast et al., 2013). The database was manually curated to extend the taxonomic outline of all 183 relevant lineages to genus level by linking the taxonomy to the termite-specific groups to that of 184 the DictDb v3 database (Mikaelyan et al., 2015b). 16S rRNA gene sequences contained in the 185 MAGs were aligned with SINA version 1.2.11 (Pruesse, Peplies \& Glöckner, 2012) and 186 imported into the reference database. Sequences longer than $>100 \mathrm{bp}$ were added to the 187 reference trees using the parsimony tool of ARB version 6.0.6 (Ludwig et al., 2004). If none of 188 the MAGs in a cluster contained a 16S rRNA gene longer than $100 \mathrm{bp}$, or if the placement of the 189 16S rRNA genes in the bin conflicted with the results of the phylogenomic analysis (indicating a 190 contamination), the phylogenomic classification was used.

\section{Estimation of the relative abundance of the MAGs in each metagenome}

193 For each metagenome, raw reads were mapped against MAGs using BWA (Li \& Durbin, 2009) 194 with default parameters. Unmapped reads and reads mapped to more than one location were 195 removed by using SAMtools (Li et al., 2009) with parameters: F 0x904. Reads mapped to each 196 MAGs were summarized using the "pileup.sh" script (BBmap 38.26) (Bushnell, 2014). The 197 relative abundance of each MAG was calculated as the total number of reads mapped to a MAG 198 divided by the total number of reads in the corresponding metagenome sample, as described in 199 Hua et al., 2019. Similarly, the MAG coverage was estimated by multiplying the mapped reads 200 by the read length and dividing it by the MAG length.

\section{Statistical analyses}

Statistical analyses were performed with R version 3.4.4 (R Development Core Team, 2019), and data were visualized with the ggplot2 (Wickham, 2016) package. Correlations between quantitative variables were investigated with Pearson's product moment correlation coefficient.

\section{Results and Discussion}

\section{Metagenomes and MAGs overview}

Metagenomic reads were generated from the P1, P3 and P4 proctodeal compartments of the gut of the two termite species Embiratermes neotenicus and Labiotermes labralis. These six metagenomes were combined with 24 previously published metagenomes from the gut of higher

212 termites (Rossmassler et al., 2015) in order to obtain data encompassing different gut 
213 compartments from eight species of higher termites feeding on different lignocellulosic

214 substrates ranging from wood to soil (Table 1). Metagenomic binning of these 30 termite gut 215 metagenomes yielded 1732 bins in total (Table S1). For further analysis, we selected only those 216 bins that represented high-quality ( 135 bins, $>90 \%$ complete and $<5 \%$ contamination) and 217 medium-quality (454 bins, $>50 \%$ complete and $<10 \%$ contamination) MAGs (Table 1 , Table 218 S1). The present study focused on these 589 MAGs, which showed on average a 38.6-fold 219 coverage (Table S2).

220 The number of MAGs recovered from the different metagenomes did not show a Gaussian distribution. Instead, we found a significant and positive relationship between the number of metagenome-assembled reads and the number of MAGs recovered $(r=0.85$, $p<0.0001$ ), indicating that assembly success and sequencing depth were important predictors of genome reconstruction success (Figure 1). This is in agreement with benchmarking reports on metagenomic datasets (Sczyrba et al., 2017) and underscore that a good quality assembly is a prerequisite for high binning recovery, which is important to consider when designing a metagenomic project for the purpose of binning. A significantly higher number of assembled reads and of MAGs recovered was observed in the current dataset compared to the Rossmassler et al., 2015 dataset (Wilcoxon test, $p<0.005$ ), highlighting the importance of this new dataset (Figure 1).

\section{MAGs taxonomy and abundance}

We investigated the phylogenomic context of the 589 MAGs. An initial automated classification of the MAGs using CheckM and when available, the taxonomic assignment of the 16S rRNA gene, identified representatives of 15 prokaryotic phyla (Table S3). Initially, 142 MAGs (24\% of the dataset) remained unclassified at the phylum level, and key taxa of the termite microbiota, such as Fibrobacteres and Treponema, were absent or only poorly represented. This is partly explained by the lack of representative genomes for certain taxa in the reference genome tree provided in the current version of CheckM (e.g., only one Fibrobacteres genome and one Elusimicrobia genome, and an absence of Bathyarchaeota and Kiritimatiellaeota genomes). New tools incorporating larger databases, such as GTDB-Tk (Parks et al., 2018; Chaumeil et al., 2019), will probably resolve such issues.

We improved the taxonomic resolution of the classification by iteratively constructing genome trees for each clade of interest that included all recently published reference genomes. This approach allowed the successful classification of all 589 MAGs, in some cases down to the genus level (Table S2). Thirty-eight MAGs were from the archaeal domain, and 551 MAGs were from the bacterial domain, which together represented a total of 17 prokaryotic phyla (Figure 2).

247 Obvious patterns in the taxonomic distribution of the MAGs according to the sample origin were not apparent, which reflects the lack of effects of the gut compartments and/or of the diet of the host on the genome taxonomy (Figures S1 and S2). Among the most abundant phyla, genomes 
250

251

252

253

254

255

256

257

258

259

260

261

262

263

264

265

266

267

268

269

270

271

272

273

274

275

276

277

278

279

280

281

282

283

284

285

286

287

were recovered from different gut compartments and diets, indicating no sample specificity for the major phyla and thus, suggesting a good coverage of the diversity among gut compartments and host diets.

The MAG taxonomy was further refined by placing all $16 \mathrm{~S}$ rRNA genes recovered from the bins into the phylogenetic framework of the current SILVA reference database, which allowed classifying most of the MAGs down to genus level (Table S2). When we compared the taxa represented by the MAGs to the distribution of the corresponding taxa in amplicon libraries of the bacterial gut microbiota of a representative selection of higher termites that were classified using the same framework (Lampert, Mikaelyan \& Brune, 2019), we found a high level of congruence between the datasets. The MAGs represented 15 of the 19 bacterial phyla in the amplicon libraries that comprised $>0.1 \%$ of all reads, including all core phyla (represented in $>80 \%$ of all host species) with the exception of Verrucomicrobia (Figure 3). A high representation in the amplicon libraries of the taxa represented by MAGs was confirmed at all taxonomic ranks down to the genus level (Table S4), underscoring that the present dataset covers the majority of lineages that colonize the higher-termite gut.

We computed the relative abundance of each MAG. These abundances ranged from $0.005 \%$ to $4.03 \%$ (Table S2), with a mean value of $0.19 \%$. These values indicated that the present dataset includes major taxonomic groups of the termite gut microbiota, which was confirmed when we looked at the taxonomic distribution of the MAGs. Considering the MAG relative abundance and not only their presence within samples, we could observe an effect of the host diet on the taxonomic distribution (Figure 4). Indeed, similarities were observed when we compared taxonomic patterns of the MAG relative abundance with previously published 16S rRNA gene amplicon-based surveys (Abdul Rahman et al., 2015; Mikaelyan, Meuser \& Brune, 2017). For instance, Spirochaetes were the most abundant phylum within the wood-feeding termite Nasutitermes corniger, and their proportion decreases along the humification gradient, being less abundant in the gut of humus feeders and litter feeders and even less abundant in soil feeders, in the favor of other phyla such as Firmicutes. Fibrobacteres were preferentially abundant within wood- and litter-feeder samples (Figure 4). Interestingly, a significant and negative relationship between the number of metagenome-assembled reads in a sample and the MAG relative abundances within this sample $(r=-0.33, p<0.0001)$ was observed across all the samples. This could be partly explained by the fact that increasing sequencing depth would increase the number of metagenome-assembled reads and thus allow the binning of sequences from less abundant organisms. However, since quantity of metagenome-assembled reads and relative abundance are not independent variables, it also implies that MAG relative abundances can not be directly quantitatively compared between samples but only within a single sample. Thus, proportions of taxa within a sample using relative abundance can be used to describe such sample.

\section{Archaea}

The archaeal domain was represented by members of the phyla Euryarchaeota and 
288 Bathyarchaeota (Figure 5, Figure S3). Euryarchaeota were represented by 23 MAGs that were 289 classified as members of the genera Methanobrevibacter (family Methanobacteriaceae; 3 290 MAGs) and, Methanimicrococcus (family Methanosarcinaceae; 3 MAGs), and members of the 291 family Methanomethylophilaceae (16 MAGs), nine of them in the genus Candidatus 292 Methanoplasma. MAGs assigned as Euryarchaeota encompassed three (Methanobacteriales, 293 Methanosarcinales, and Methanomassiliicoccales) of the four orders of methanogens found in 294 termite guts (Brune, 2018); Methanomicrobiales were absent from the present dataset. This 295 genomic resource will be extremely valuable for a better understanding of the genomic basis of 296

297

298

299

300

301

302

303

304

305

306

307

308

309

310

311

312

313

314

315

316

317

318

319

320

321

322

323

324

325 methanogenesis in the termite gut and more generally for investigating the functional role of archaea in arthropod guts. Indeed, Euryarchaota have been found to be present in virtually all termite species investigated (Brune, 2018), and a global 16S rRNA gene survey has revealed that this phylum is the most abundant archaeal clade in arthropod-associated microbiota (Schloss et al., 2016). Bathyarchaeota were represented by 15 MAGs, which formed a termite-specific cluster, with Bathyarchaeota reconstructed from sediments of the White Oak River (WOR) estuary (North Carolina, USA) as next relatives (Lazar et al., 2016) (Figure 5). Bathyarchaeota is a lineage formerly known as Miscellaneous Crenarchaeota Group (MCG), which has been reported to occur in the gut of soil-feeding termites (Friedrich et al., 2001). To date, MAGs of Bathyarchaeota have been mostly derived from aquatic environments (Zhou et al., 2018). Here, we identify the members of this lineage as Bathyarchaeota and provide the first genomes from this environment. Interestingly, Bathyarchaeota MAGs were particularly abundant in humus-, litter- and soil-feeding termites (Figure 4); a genomic characterization, combined with analyses of their abundance and localization, should shed light on the metabolic potential of these organisms and their functional role in termite guts.

\section{Firmicutes}

Firmicutes was by far the phylum with the highest number of MAGs, but also the phylum with the highest average relative abundance (33.5\%) in 16S rRNA gene-based surveys (Figure 3 ). The 237 MAGs (40\% of the total dataset) represented three classes (Bacilli, Clostridia and Erysipelotrichia) and ten families, including four members of Streptococcaceae (Bacilli) and three members of Turicibacteraceae (Erysipelotrichia). Clostridia was the most diverse and rich class (229 MAGs), in which Ruminococcaceae (95 MAGs), Defluviitaleaceae (67 MAGs), Lachnospiraceae (4 MAGs), Peptococcaceae (16 MAGs), Christensenellaceae (9 MAGs), Eubacteriaceae (2 MAGs), Family XIII incertae sedis (6 MAGs) and Clostridiales vadinBB60 group (22 MAGs) families were identified. These high numbers of Ruminococcaceae and Defluviitaleaceae MAGs were reflected by high relative abundances of these two families in $16 \mathrm{~S}$ rRNA gene-based surveys (15.9\% and 3.1\% for the Ruminococcaceae and Defluviitaleaceae, respectively; Table S4). Interestingly, among the Defluviitaleaceae, the genomes were mainly recovered from the P1 compartment (53 MAGs, i.e., 79\% of the family members) whereas Ruminococcaceae were predominantly recovered from the P3 compartment (59 MAGs, i.e., 62\% 
326 of the family members). Further studies should investigate the potential metabolic specialization 327 of these two families in relation to the gut physicochemical properties. A fourth class-level 328 lineage could not be further classified for lack of reference genomes. In a recent global $16 \mathrm{~S}$ 329 rRNA gene-based survey, it has been suggested that many novel lineages of Firmicutes in insect330 associated metagenomes are hidden (Schulz et al., 2017). Our present study confirms this idea 331 but our genome trees also provide evidence of new lineages. Here we report the first genomes of 332 uncultured termite-specific lineages (Table S4) that were already detected in previous 16S rRNA gene-based surveys (Bourguignon et al., 2018). For example, the phylogenomic tree of the most abundant family Ruminococcaceae (Figure S4) showed various termite-specific clusters, including a cluster of 18 MAGs closely related to Sporobacter termitidis isolated from Nasutitermes lujae (Grech-Mora et al., 1996). Lachnospiraceae, Ruminococcaceae, Turicibacteraceae (previously classified as Erysipelotrichaceae), and Defluviitaleaceae (previously classified as Lachnospiraceae) have been reported among the dominant taxa in termite guts (Mikaelyan, Meuser \& Brune, 2017), but most of them remain uncultivated and/or with few representative genomes. As such, many questions regarding their ecology and metabolism remain open. With 237 Firmicutes MAGs recovered from different gut compartments and from hosts with different diets, the present study provides the material for further genomic exploration of the role of these bacteria in plant polysaccharide degradation, based for instance on CAZyme distribution (Lombard et al., 2014). Since diet has been shown to be the main factor shaping gut community composition in higher termites (Mikaelyan et al., 2015a), one might hypothesize the existence of different arsenals of lignocellulolytic enzymes, potentially reflecting the host diet specificity (balance between cellulose, lignin, and hemicelluloses). More generally, Firmicutes and especially Ruminococcaceae are also abundant and metabolically important in rumen systems (Svartström et al., 2017; Söllinger et al., 2018; Stewart et al., 2018). At a broader scale, our dataset will allow comparative studies between intestinal tract microbiota of ruminants and phytophagous or xylophagous invertebrates, which would allow a better understanding of plant polysaccharide degradation across the tree of life.

\section{Actinobacteria}

Actinobacteria was the second most abundant phylum with 71 MAGs, including members of the classes Acidimicrobiia, Actinobacteriia, Coriobacteriia and Thermoleophilia (Figure S5). Eleven families were represented, namely Propionibacteriaceae (12 MAGs), Promicromonosporaceae (3 MAGs), Clostridiales incertae sedis (16 MAGs), OPB41 (16 MAGs) Cellulomonadaceae (7 MAGs), Frankiaceae (1 MAG), Sanguibacteraceae (4 MAGs), Microbacteriaceae (2 MAGs), Nocardioidaceae (2 MAGs), Acidimicrobiaceae (1 MAG), Nocardiaceae (1 MAG) and Conexibacteraceae (1 MAG). Among these 71 MAGs, 36 were recovered from humus feeders, 33 from soil feeders but only 2 from wood feeders, which suggests a higher prevalence in

362 termites with a more humified diet. This phylum is known to be present and of significant 363 abundance in both the nest (Sujada, Sungthong \& Lumyong, 2014) and gut of termites (Le Roes- 
364 Hill, Rohland \& Burton, 2011), but to be more abundant in the nest (Moreira et al., 2018). This 365 was for instance the case for the families Acidimicrobiaceae, Nocardiaceae, 366 Promicromonosporaceae, Microbacteriaceae, Nocardioidaceae, and Propionibacteriaceae,

367 which were more abundant in the nest than in the gut of workers or soldiers of Procornitermes 368 araujoi (Moreira et al., 2018). Therefore, one of the key questions regarding this phylum 369 concerns their effective role in lignocellulose degradation in the termite guts. Are they just 370 present in the surrounding environment of the termite and thus sometimes transit from the gut or 371 are they actively involved in food digestion? The MAGs obtained in the present study will allow 372 to address such questions by evaluating gene expression of these organisms using 373 metatranscriptomic data from higher termites (He et al., 2013; Marynowska et al., 2017).

374

375

376

377

378

379

380

381

382

383

384

385

386

387

388

389

390

391

392

393

394

395

396

397

398

399

400

\section{Spirochaetes}

The phylum Spirochaetes was represented by 68 MAGs from wood-, soil-, litter- and humusfeeding termites. It has long been known that Spirochaetes are a diverse and important lineage in termite gut (Paster et al., 1996; Lilburn, Schmidt \& Breznak, 1999), especially because of their involvement in reductive acetogenesis (Leadbetter et al., 1999; Ohkuma et al., 2015) and in hemicellulose degradation (Tokuda et al., 2018). In terms of abundance, Spirochaetes are among the dominant phyla in termite guts (Figure 3) and may represent more than half of the bacterial relative abundance in some species (Diouf et al., 2018a). Three Spirochaetes orders, namely Brevinematales (1 MAG), Leptospirales (4 MAGs) and Spirochaetales (59 MAGs), were identified (Figure 6, Figure S6). In the latter order, 54 MAGs recovered from the P1, P3 and P4 compartments of wood-, litter-, humus-, and soil-feeding hosts were assigned to the termitespecific cluster Treponema I (Ohkuma, Iida \& Kudo, 1999; Lilburn, Schmidt \& Breznak, 1999) and represent the first genomes of this cluster from higher termites. Indeed, to date only two Treponema I genomes are available, and both were recovered from isolates, namely T. azotonutricium and T.primitia, from the hindgut of the lower termite Zootermopsis angusticollis (Graber, Leadbetter \& Breznak, 2004). Thus, our dataset significantly expands the genomic resources for this taxonomic group. Subclusters of this clade have been identified on a dedicated genome tree (Figure 6). The genome tree topology is in agreement with a previous phylogenomic Spirochaetes study (Gupta, Mahmood \& Adeolu, 2013). Regarding Spirochaetes classification, our tree topology suggests that the genus Treponema could be elevated at least to the family rank due to the presence of distinct Treponema clusters (Figure 6). This observation is also in agreement with the recent Genome Taxonomy Database, which now proposes a Treponemataceae family and a Treponematales order (Parks et al., 2018; Chaumeil et al., 2019).

\section{Fibrobacteres}

Members of the phylum Fibrobacteres are abundant in the hindgut of wood-feeding higher termites (Figure 3) (Hongoh et al., 2006), where they have been identified as fiber-associated cellulolytic bacteria (Mikaelyan et al., 2014). Here, thirteen members of the Fibrobacteres

Peer) reviewing PDF | (2019:08:40743:1:1:NEW 19 Dec 2019) 
401 phylum were recovered from the P1, P3, and P4 compartments of wood-, litter-, humus-, and 402 soil-feeding termites. These genomes encompass the 3 orders, namely Chitinispirillales (Sorokin 403 et al., 2016), previously known as TG3 subphylum 1 (Hongoh et al., 2006, 5 MAGs), 404 Chitinivibrionales (previously known as TG3 subphylum 2; 2 MAGs), and Fibrobacterales (6 405 MAGs). While a previous study of termites guts had already provided MAGs of 406 Chitinivibrionaceae and Fibrobacteraceae and documented their fiber-degrading capacities 407 (Abdul Rahman et al., 2016), the present study provides the five first genomes of the termite408 associated members of Chitinispirillaceae (Figure 7).

409 Phylogenomic analysis indicates that the MAGs classified as Fibrobacterales represent a 410 termite-specific cluster among Fibrobacteraceae that comprises Candidatus Fibromonas 411 termitidis and forms a sister group to the genus Fibrobacter (Figure 7, Figure S7). This is in 412 agreement with a previous study that identified the same lineage (but classified as family 413 Fibromonadaceae) by 16S rRNA gene-based and phylogenomic analyses (Abdul Rahman et al., 414 2016). None of the MAGs fell into the genus Fibrobacter, which was absent also in all 16S 415 rRNA gene-based surveys of termite gut microbiota (Hongoh et al., 2006; Mikaelyan et al., 416 2015b; Bourguignon et al., 2018). Members of this genus have been isolated from the 417 gastrointestinal tracts of mammals and bird herbivores (Neumann, McCormick \& Suen, 2017), 418 where they are potentially involved in cellulose and hemicellulose degradation (Neumann \& 419 Suen, 2018). This suggests co-evolutionary patterns among different Fibrobacteres clades within 420 animal hosts with a lignocellulose-based diet.

\section{Proteobacteria and Bacteroidetes}

422 Sixty-seven MAGs of Proteobacteria belonging to Alphaproteobacteria (23 MAGs), 423 Gammaproteobacteria (20 MAGs), and Deltaproteobacteria (24 MAGs) were recovered from 424 all hindgut compartments of litter-, humus-, and soil-feeding termites. Among the 425 Deltaproteobacteria, seven orders were identified, namely Desulfobacterales (4 MAGs, all 426 assigned to Desulfobulbus), Desulfovibrionales (5 MAGs, all Desulfovibrionaceae), 427 Desulfuromonadales (1 MAG), Myxococcales (5 Myxococcaceae and 4 Polyangiaceae), 428 Adiutricales (1 MAG), Syntrophobacterales (1 MAG) and MBNT15 group (2 MAGs). 429 Desulfovibrionaceae (Desulfovibrionales) members of gut and termite-gut clusters have been 430 found to be highly prevalent in termite guts (Bourguignon et al., 2018). Similarly, we identified 3 431 Desulfovibrionaceae MAGs that form a monophyletic clade and 2 Desulfovibrionaceae MAGs 432 that fall into a cluster of gut-associated genomes (Figure S8). This family, among others, is 433 composed of various sulfate-reducing bacteria; this functional group has already been identified 434 in different termite species (Kuhnigk et al., 1996). Thus, these MAGs could provide new 435 genomic resources to further investigate this metabolism in the termite gut.

436 Our dataset comprises 33 MAGs of Bacteroidetes (Figure S9), including members of the

437 families Cluster V (4 MAGs), RC9 gut group (6 MAGs), Paludibacteraceae (2 MAGs, both 
438

439

440

441

442

443

444

445

446

447

448

449

450

451

452

453

454

455

456

457

458

459

460

461

462

463

464

465

466

467

468

469

470

471

472

473

474

475

assigned to the Paludibacter genus), Rikenellaceae (9 MAGs), Marinilabiliaceae (1 MAG), and Prolixibacteraceae (1 MAG). These Bacteroidetes were found in the P1, P3, and P4 compartments and in wood-, litter-, humus-, and soil-feeding termites. In Blattodea guts, different clusters of Alistipes (Bacteroidetes) have been found in a 16S rRNA gene survey (Mikaelyan et al., 2015b). Two MAGs from Labiotermes labralis belonging to the Rikenellaceae family and closely related to Alistipes have been identified. Additionally, among Bacteroidetes, four MAGs, all originating from $\mathrm{P} 4$ compartments, fall into the Cluster V family that contains symbionts of flagellates from guts of lower termites (Hongoh et al., 2008b; Yuki et al., 2015). We also recovered two MAGs assigned to Paludibacter; Paludibacter propionicigenes and Paludibacter jiangxiensis are both strictly anaerobic, propionate-producing bacteria isolated from rice paddy field (Ueki et al., 2006; Qiu et al., 2014). Propionate is produced by fermenting bacteria in the gut of termites (Odelson \& Breznak, 1983); these bacteria utilize glucose generated by cellulose degradation to form succinate and propionate (Tokuda et al., 2014). $P$. propionicigenes might be involved in nitrogen fixation, as $n i f H$ transcripts assigned to this species are the most abundant in the gut of the wood-feeding beetle Odontotaenius disjunctus (Ceja-Navarro et al., 2014).

\section{Saccharibacteria, Synergistetes and Planctomycetes}

Fifteen MAGs of Candidatus Saccharibacteria (also known as candidate division TM7) were reconstructed from the P1, P3, and P4 compartments of wood-, litter-, humus- and soil-feeding termites (Figure S10). Most of them originated from humus feeders (11 MAGs), especially from the P3 compartment (8 out of these 11 MAGs). Similarly, six MAGs of Synergistetes, all belonging to the Synergistaceae family that contains a termite/cockroach cluster (Mikaelyan et al., 2015b), were recovered from the P3 and P4 compartments of humus- and soil-feeding termites (Figure S11). Both Saccharibacteria and Synergistetes were recently highlighted as numerically important clades of the termite gut microbiota, with some OTUs being present in the gut of the majority of 94 termite species collected across four continents (Bourguignon et al., 2018). They were also contributing to the core microbiota of higher termites (Figure 3). Genomic analysis of these MAGs should help in understanding the roles of these bacteria in termite gut and also provide clues for designing successful isolation media to study their physiology.

Twelve MAGs were assigned to the phylum Planctomycetes, including 4 to the class Phycisphaerae (and among them 2 classified as Tepidisphaerales CPla-3 termite group), one to class vadinHA49 and 7 to the class Planctomycetia (all classified as Pirellulaceae) (Figure S12). These MAGs were recovered from the P3, P4, and P5 compartments and were restricted to humus- and soil-feeding termites. The recovery of Planctomycetes was expected, especially from the Pirellulaceae family, which also contains termite / cockroach clusters (Mikaelyan et al., 2015b). Interestingly, we found three MAGs from the P4 and P5 compartments of Cubitermes ugandensis, with one 16S rRNA gene sequence assigned to the Rs-B01 termite group, described in a previous study investigating the gut microbiota of the same termite species (Köhler et al., 
476 2008). When such $16 \mathrm{~S}$ rRNA gene information is available, it will allow the direct linkage 477 between prokaryotic taxonomy and potential metabolisms.

\section{$478 \quad$ Other phyla}

479

480

481

482

483

484

485

486

487

488

489

490

491

492

493

494

495

496

497

498

499

500

501

502

503

504

505

506

507

508

509

510

511

512

Nine members of the phylum Elusimicrobia were identified, including members of the class Endomicrobia ( 8 members) and Elusimicrobia (1 member) (Figure S13). These were found in all hindgut compartments and were restricted to humus- and soil-feeding termites. Currently, only three complete genomes of Elusimicrobia from insect guts are available: Elusimicrobium minutum from the gut of a humivorous scarab beetle larva (Herlemann et al., 2009), and Endomicrobium proavitum (Zheng \& Brune, 2015) and Candidatus Endomicrobium trichonymphae (Hongoh et al., 2008a) from the termite gut. Here, we provided 9 additional genomes from the guts of humus- and soil-feeding termites.

The Chloroflexi phylum was represented by eight MAGs (all Dehalococcoidia), including seven belonging to the family Dehalococcoidaceae and one to the family Dehalobiaceae, found exclusively in the P3 and P4 compartments of humus- and soil-feeding termites (Figure S10). Their function in termite gut remains unclear, but Chloroflexi, including Dehalococcoidia, were found to be enriched in lignin-amended tropical forest soil (DeAngelis et al., 2011), where oxygen concentration and redox potential are highly variable, as in the termite gut (Brune, 2014). Therefore, their ability to use oxygen as final electron acceptor and their potential involvement in lignin degradation could be investigated by comparative genomics.

Minor phyla were also present in our dataset. Two MAGs assigned as Cloacimonetes (Figure S14) and five MAGs assigned as Kiritimatiellaeota were recovered from the P3 compartment of the two humus-feeding termites Neocapritermes taracua and Termes hospes (Figure S15). Kiritimatiellaeota have been reported to be present in the digestive tract of various animals (Spring et al., 2016). The few clones obtained from termite guts, which had been tentatively classified as uncultured Verrucomicrobia, were mostly obtained with planctomycetespecific primers (Köhler et al., 2008), underscoring the potential biases in amplicon-based studies toward certain taxa. Similarly, one MAG of Microgenomates (also known as candidate division OP11), which probably represents a lineage of Pacebacteria that was discovered only in a recent amplicon-based analysis but occurs in the majority of termites investigated (Bourguignon et al., 2018), was reconstructed from the P3 compartment of Termes hospes (Figure S10).

Finally, four MAGs classified as Acidobacteria were reconstructed from either the P3 or P4 compartments of humus- and soil-feeding termites (Figure S16), which show a moderately alkaline or circumneutral $\mathrm{pH}$ in comparison to the highly alkaline P1. Of these four genomes, two were assigned to the M1PL1-36 termite group within the family Holophagaceae and one to the Acidobacteriaceae family. Acidobacteria can represent a significant fraction of the termite gut microbiota, especially in wood-feeding termites (Hongoh et al., 2005; Wang et al., 2016; 
513 Bourguignon et al., 2018). In the gut of higher termites, this phylum is present in the core

514 microbiota (Figure 3). Moreover, Holophagaceae and Acidobacteriaceae have been reported to

515 be present in moderately acidic lignocellulosic substrates, such as peatland soil (Schmidt et al., 516 2015) and decaying wood (Hervé et al., 2014). Genomic analysis should help us in identifying 517 the metabolic potential of these MAGs for lignocellulose degradation.

$518 \quad$ Phyla not represented by MAGs

519 Several bacterial phyla and one archaeal phylum containing prominent taxa that have been 520 identified in previous 16S rRNA gene surveys of termite guts were not represented among the 521 MAGs recovered in the present study. They include Cyanobacteria (class Melainabacteria; 522 Utami et al., 2018), Lentisphaerae (Köhler et al., 2012; Sabree \& Moran, 2014), 523 Verrucomicrobia (Wertz et al., 2012), and Thaumarchaeota (Friedrich et al., 2001; Shi et al., 524 2015). Also intracellular symbionts of termite tissues, such as Wolbachia (Proteobacteria) (Diouf 525 et al., 2018b) were not recovered. Possible reasons are a low relative abundance and/or a high 526 phylogenetic diversity of the respective lineages. Although larger metagenomes should improve 527 the chances of their recovery in the medium- and high-quality bins, targeted single-cell based 528 approaches have proven to be quite effective in recovering these genomes (Ohkuma et al., 2015; 529 Yuki et al., 2015; Utami et al., 2019).

530

\section{Conclusions}

532 The 589 MAGs reported here represent the largest genomic resource for arthropod-associated 533 microorganisms available to date. We recovered representatives of almost all major prokaryotic 534 lineages previously identified in 16S rRNA gene amplicon-based surveys of the gut of higher 535 termites from the metagenomes. This provides the foundations for studying the metabolism of 536 the prokaryotic gut microbiota of higher termites, including the key members involved in carbon 537 and nitrogen biogeochemical cycles, and important clues that may help in cultivating 538 representatives of these understudied clades.

\section{Acknowledgments}

The work conducted by the U.S. Department of Energy Joint Genome Institute, a DOE Office of

542 Science User Facility, is supported by the Office of Science of the U.S. Department of Energy under Contract No. DE-AC02-05CH11231. The authors thank all JGI staff, particularly their project manager Tijana Glavina del Rio, for their excellent service. The technical assistance of

545 Katja Meuser is highly appreciated. We also thank the three anonymous reviewers and the editor 546 for their helpful and constructive comments. 
550 Table 1. Recovery of metagenome-assembled genomes (MAGs) from the 30 termite gut 551 metagenomes analyzed in this study. The host termite, its mitochondrial genome accession 552 number, dietary preference, and the originating gut compartments are indicated. $C$ crop (foregut), $553 M$ midgut, P1-P5 proctodeal compartments (hindgut). The sample codes used for the 554 metagenomes are the combination of host ID and gut compartment.

\section{Figure legends}

557

558

559

560

561

562

563

564

565

566

567

568

569

570

571

572

573

574

575

576

577

578

579

580

581

Figure 1: Relationship between the number of MAGs recovered and the number of assembled reads in the respective metagenomes. The linear regression line and the Pearson correlation coefficient ( $r$ ) are shown for the entire dataset.

Figure 2: Distribution of the 589 MAGs among bacterial and archaeal phyla. This maximum-likelihood tree was inferred from a concatenated alignment (amino acids) of 43 protein-coding genes $(6,801$ positions) using the $\mathrm{LG}+\mathrm{G}+\mathrm{I}$ model of evolution.

Figure 3: Phylum-level representation of MAGs among the bacterial gut microbiota of higher termites. The average abundance of the corresponding lineages in 16S rRNA amplicon libraries of higher termites from different diet groups is shown for comparison. Core lineages represented in at least $80 \%$ of these samples are marked. For an interactive spreadsheet resolving each lineage to genus level, see Supplementary Information (Table S4).

Figure 4: Relative abundance of the MAGs from different phyla among the respective metagenomes. Circle size indicates the relative abundance of the MAGs among the respective metagenome sample; color indicates host diet. To estimate the relative abundance of each MAG, the total number of reads mapped to a MAG was divided by the total number of reads in the corresponding metagenome sample.

Figure 5: Phylogenomic tree of the archaeal domain. This maximum-likelihood tree was inferred from a concatenated alignment of 43 proteins $(6,682$ positions) using the $\mathrm{LG}+\mathrm{G}+\mathrm{I}+\mathrm{F}$ model of amino-acid evolution. Branch supports were calculated using a Chi2-based parametric approximate likelihood-ratio test. Names in bold included MAGs recovered in the present study. Clusters shaded in brown consist exclusively of MAGs from termite guts and clusters shaded in gray contain genomes from termite guts. The Asgard group was used as outgroup.

Figure 6: Phylogenomic tree of the Spirochaetes phylum. This maximum-likelihood tree was inferred from a concatenated alignment of 43 proteins $(6,741$ positions) using the $\mathrm{LG}+\mathrm{G}+\mathrm{I}+\mathrm{F}$ model of amino-acid evolution. Branch supports were calculated using a Chi2-based parametric 
582 approximate likelihood-ratio test. Names in bold included MAGs recovered in the present study.

583 Clusters shaded in brown consist exclusively of genomes from termite guts and clusters shaded

584 in gray contain genomes from termite guts. Elusimicrobia and Cyanobacteria were used as 585 outgroup.

586 Figure 7: Phylogenomic tree of the Fibrobacteres phylum. This maximum-likelihood tree was 587 inferred from a concatenated alignment of 43 proteins (6,516 positions) using the $\mathrm{LG}+\mathrm{G}+\mathrm{I}+\mathrm{F}$

588 model of amino-acid evolution. Branch supports were calculated using a Chi2-based parametric 589 approximate likelihood-ratio test. Names in bold included MAGs recovered in the present study. 590 Clusters shaded in brown consist exclusively of genomes from termite guts and clusters shaded 591 in gray contain genomes from termite guts. Bacteroidetes were used as outgroup.

592

593

594 Supplementary information

595 Supplementary Table S1: Metagenome characteristics.

596 Supplementary Table S2: Final taxonomic assignment and characteristics of the MAGs

597 Supplementary Table S3: Initial taxonomic assignment of the MAGs

598 Supplementary Table S4: Representation of the MAGs among the bacterial gut microbiota of 599 higher termites at different taxonomic ranks

600 Figure S1: Phylogenomic distribution of the MAGs according to the host diet. The outer 601 rings show the occurrence of MAGs in termites with different diets. The maximum likelihood 602 tree was inferred from a concatenated alignment of 43 proteins $(6,801$ positions) using the $603 \mathrm{LG}+\mathrm{G}+\mathrm{I}$ model of amino-acid evolution.

604 Figure S2: Phylogenomic distribution of the MAGs according to the gut compartment of 605 the host. The outer rings show the occurrence of MAGs in the different termite gut 606 compartments: $C$ crop (foregut), $M$ midgut, $P 1-P 5$ proctodeal compartments (hindgut). The 607 maximum-likelihood tree was inferred from a concatenated alignment of 43 proteins $(6,801$ 608 positions) using the $\mathrm{LG}+\mathrm{G}+\mathrm{I}$ model of amino-acid evolution.

609 Figure S3: Phylogenomic tree of the Archaea. This maximum-likelihood tree was inferred 610 from a concatenated alignment of 43 proteins $(6,682$ positions) using the $\mathrm{LG}+\mathrm{G}+\mathrm{I}+\mathrm{F}$ model of 611 amino-acid evolution. Branch supports were calculated using a Chi2-based parametric 612 approximate likelihood-ratio test. Asgard group was used as outgroup. Names in bold included 613 MAGs recovered in the present study.

614 Figure S4: Phylogenomic tree of the Ruminococcaceae family (Firmicutes). This maximum615 likelihood tree was inferred from a concatenated alignment of 43 proteins (6,585 positions) using 
616 the LG+G+I model of amino-acid evolution. Branch supports were calculated using a Chi2617 based parametric approximate likelihood-ratio test. Dorea and Butyrivibrio (Lachnospiraceae)

618 species were used as outgroup. Names in bold included MAGs recovered in the present study.

619 Figure S5: Phylogenomic tree of the Actinobacteria. This maximum-likelihood tree was 620 inferred from a concatenated alignment of 43 proteins (6,611 positions) using the $\mathrm{LG}+\mathrm{G}+\mathrm{I}+\mathrm{F}$ 621 model of amino-acid evolution. Branch supports were calculated using a Chi2-based parametric 622 approximate likelihood-ratio test. Chloroflexi species were used as outgroup. Names in bold 623 included MAGs recovered in the present study.

624 Figure S6: Phylogenomic tree of the Spirochaetes. This maximum-likelihood tree was inferred 625 from a concatenated alignment of 43 proteins (6,741 positions) using the $\mathrm{LG}+\mathrm{G}+\mathrm{I}+\mathrm{F}$ model of 626 amino-acid evolution. Branch supports were calculated using a Chi2-based parametric 627 approximate likelihood-ratio test. Elusimicrobia and Cyanobacteria were used as outgroup. 628 Names in bold included MAGs recovered in the present study.

629 Figure S7: Phylogenomic tree of the Fibrobacteres. This maximum-likelihood tree was 630 inferred from a concatenated alignment of 43 proteins (6,516 positions) using the $L G+G+I+F$ 631 model of amino-acid evolution. Branch supports were calculated using a Chi2-based parametric 632 approximate likelihood-ratio test. Bacteroidetes were used as outgroup. Names in bold included 633 MAGs recovered in the present study.

634 Figure S8: Phylogenomic tree of the Desulfovibrionaceae family (Deltaproteobacteria). This 635 maximum-likelihood tree was inferred from a concatenated alignment of 43 proteins $(6,602$ 636 positions) using the $\mathrm{LG}+\mathrm{G}+\mathrm{I}$ model of amino-acid evolution. Branch supports were calculated 637 using a Chi2-based parametric approximate likelihood-ratio test. Desulfonatronum species were 638 used as outgroup. Names in bold included MAGs recovered in the present study.

639 Figure S9: Phylogenomic tree of the Bacteroidetes. This maximum-likelihood tree was 640 inferred from a concatenated alignment of 43 proteins (6,645 positions) using the $\mathrm{LG}+\mathrm{G}+\mathrm{I}$ 641 model of amino-acid evolution. Branch supports were calculated using a Chi2-based parametric 642 approximate likelihood-ratio test. Chlorobi species were used as outgroup. Names in bold 643 included MAGs recovered in the present study.

644 Figure S10: Phylogenomic tree of the Chloroflexi, Saccharibacteria and Microgenomates. 645 This maximum-likelihood tree was inferred from a concatenated alignment of 43 proteins $(6,466$ 646 positions) using the $\mathrm{LG}+\mathrm{G}+\mathrm{I}+\mathrm{F}$ model of amino-acid evolution. Branch supports were calculated 647 using a Chi2-based parametric approximate likelihood-ratio test. Actinobacteria species were 648 used as outgroup. Names in bold included MAGs recovered in the present study.

649 Figure S11: Phylogenomic tree of the Synergistetes. This maximum-likelihood tree was 650 inferred from a concatenated alignment of 43 proteins (6,429 positions) using the $\mathrm{LG}+\mathrm{G}+\mathrm{I}+\mathrm{F}$ 651 model of amino-acid evolution. Branch supports were calculated using a Chi2-based parametric 
652 approximate likelihood-ratio test. Elusimicrobia species were used as outgroup. Names in bold 653 included MAGs recovered in the present study.

654 Figure S12: Phylogenomic tree of the Planctomycetes. This maximum-likelihood tree was 655 inferred from a concatenated alignment of 43 proteins (5,351 positions) using the $\mathrm{LG}+\mathrm{G}+\mathrm{I}+\mathrm{F}$ 656 model of amino-acid evolution. Branch supports were calculated using a Chi2-based parametric 657 approximate likelihood-ratio test. Verrucomicrobia species were used as outgroup. Names in 658 bold included MAGs recovered in the present study.

659 Figure S13: Phylogenomic tree of the Elusimicrobia. This maximum-likelihood tree was 660 inferred from a concatenated alignment of 43 proteins (6,399 positions) using the $\mathrm{LG}+\mathrm{G}+\mathrm{I}+\mathrm{F}$ 661 model of amino-acid evolution. Branch supports were calculated using a Chi2-based parametric 662 approximate likelihood-ratio test. Spirochaetes species were used as outgroup. Names in bold 663 included MAGs recovered in the present study.

664 Figure S14: Phylogenomic tree of the Cloacimonetes. This maximum-likelihood tree was 665 inferred from a concatenated alignment of 43 proteins (6,519 positions) using the $\mathrm{LG}+\mathrm{G}+\mathrm{I}+\mathrm{F}$ 666 model of amino-acid evolution. Branch supports were calculated using a Chi2-based parametric 667 approximate likelihood-ratio test. Fibrobacteres species were used as outgroup. Names in bold 668 included MAGs recovered in the present study.

669 Figure S15: Phylogenomic tree of the Kiritimatiellaeota. This maximum-likelihood tree was inferred from a concatenated alignment of 43 proteins (6,434 positions) using the $\mathrm{LG}+\mathrm{G}+\mathrm{I}$ model of amino-acid evolution. Branch supports were calculated using a Chi2-based parametric approximate likelihood-ratio test. Chlamydiae species were used as outgroup. Names in bold included MAGs recovered in the present study.

674 Figure S16: Phylogenomic tree of the Acidobacteria. This maximum-likelihood tree was 675 inferred from a concatenated alignment of 43 proteins (6,351 positions) using the $\mathrm{LG}+\mathrm{G}+\mathrm{I}+\mathrm{F}$ model of amino-acid evolution. Branch supports were calculated using a Chi2-based parametric approximate likelihood-ratio test. Proteobacteria species were used as outgroup. Names in bold 678 included MAGs recovered in the present study.

\section{References}

681

Abdul Rahman N., Parks DH., Vanwonterghem I., Morrison M., Tyson GW., Hugenholtz P. 2016. A phylogenomic analysis of the bacterial phylum Fibrobacteres. Frontiers in Microbiology 6. DOI: 10.3389/fmicb.2015.01469.

Abdul Rahman N., Parks DH., Willner DL., Engelbrektson AL., Goffredi SK., Warnecke F., 686

$$
\text { Scheffrahn RH., Hugenholtz P. 2015. A molecular survey of Australian and North }
$$
American termite genera indicates that vertical inheritance is the primary force shaping 
termite gut microbiomes. Microbiome 3:5. DOI: 10.1186/s40168-015-0067-8.

688

689

690

691

692

693

694

695

696

697

698

699

700

701

702

703

704

705

706

707

708

709

710

711

712

713

714

715

716

717

718

719

720

721

Albertsen M., Hugenholtz P., Skarshewski A., Nielsen KL., Tyson GW., Nielsen PH. 2013. Genome sequences of rare, uncultured bacteria obtained by differential coverage binning of multiple metagenomes. Nature Biotechnology 31:533-538. DOI: 10.1038/nbt.2579.

Anisimova M., Gascuel O. 2006. Approximate likelihood-ratio test for branches: A fast, accurate, and powerful alternative. Systematic biology 55:539-52. DOI: 10.1080/10635150600755453.

Asnicar F., Weingart G., Tickle TL., Huttenhower C., Segata N. 2015. Compact graphical representation of phylogenetic data and metadata with GraPhlAn. PeerJ 3:e1029. DOI: 10.7717/peerj.1029.

Bourguignon T., Lo N., Dietrich C., Šobotník J., Sidek S., Roisin Y., Brune A., Evans TA. 2018. Rampant host switching shaped the termite gut microbiome. Current Biology 28:649654.e2. DOI: 10.1016/j.cub.2018.01.035.

Bowers RM., Kyrpides NC., Stepanauskas R., Harmon-Smith M., Doud D., Reddy TBK., Schulz F., Jarett J., Rivers AR., Eloe-Fadrosh EA., Tringe SG., Ivanova NN., Copeland A., Clum A., Becraft ED., Malmstrom RR., Birren B., Podar M., Bork P., Weinstock GM., Garrity GM., Dodsworth JA., Yooseph S., Sutton G., Glöckner FO., Gilbert JA., Nelson WC., Hallam SJ., Jungbluth SP., Ettema TJG., Tighe S., Konstantinidis KT., Liu W-T., Baker BJ., Rattei T., Eisen JA., Hedlund B., McMahon KD., Fierer N., Knight R., Finn R., Cochrane G., Karsch-Mizrachi I., Tyson GW., Rinke C., Lapidus A., Meyer F., Yilmaz P., Parks DH., Murat Eren A., Schriml L., Banfield JF., Hugenholtz P., Woyke T. 2017. Minimum information about a single amplified genome (MISAG) and a metagenomeassembled genome (MIMAG) of bacteria and archaea. Nature Biotechnology 35:725-731. DOI: $10.1038 /$ nbt.3893.

Brune A. 2014. Symbiotic digestion of lignocellulose in termite guts. Nature Reviews Microbiology 12:168-180. DOI: 10.1038/nrmicro3182.

Brune A. 2018. Methanogenesis in the digestive tracts of insects and other arthropods. Berlin, Heidelberg, Heidelberg: Springer Berlin Heidelberg. DOI: 10.1007/978-3-540-77587-4.

Brune A., Dietrich C. 2015. The gut microbiota of termites: Digesting the diversity in the light of ecology and evolution. Annual Review of Microbiology 69:145-166. DOI:

10.1146/annurev-micro-092412-155715.

Bushnell B. 2014. BBMap: a fast, accurate, splice-aware aligner. DOI: 10.1186/1471-2105-13238.

Capella-Gutierrez S., Silla-Martinez JM., Gabaldon T., Capella-Gutiérrez S., Silla-Martínez JM., Gabaldón T. 2009. trimAl: a tool for automated alignment trimming in large-scale

Peer) reviewing PDF | (2019:08:40743:1:1:NEW 19 Dec 2019) 
phylogenetic analyses. Bioinformatics 25:1972-1973. DOI: 10.1093/bioinformatics/btp348.

723 Ceja-Navarro JA., Nguyen NH., Karaoz U., Gross SR., Herman DJ., Andersen GL., Bruns TD.,

724

725

726

727

728

729

730

731

732

733

734

735

736

737

738

739

740

741

742

743

744

745

746

747

748

749

750

751

752

753

754

755

756

Pett-Ridge J., Blackwell M., Brodie EL. 2014. Compartmentalized microbial composition, oxygen gradients and nitrogen fixation in the gut of Odontotaenius disjunctus. The ISME journal 8:6-18. DOI: 10.1038/ismej.2013.134.

Chaumeil P-A., Mussig AJ., Hugenholtz P., Parks DH. 2019. GTDB-Tk: a toolkit to classify genomes with the Genome Taxonomy Database. Bioinformatics. DOI: 10.1093/bioinformatics/btz848.

Cragg SM., Beckham GT., Bruce NC., Bugg TD., Distel DL., Dupree P., Etxabe AG., Goodell BS., Jellison J., McGeehan JE., McQueen-Mason SJ., Schnorr K., Walton PH., Watts JE., Zimmer M. 2015. Lignocellulose degradation mechanisms across the Tree of Life. Current Opinion in Chemical Biology 29:108-119. DOI: 10.1016/j.cbpa.2015.10.018.

Dahlsjö CAL., Parr CL., Malhi Y., Meir P., Chevarria OVC., Eggleton P. 2014. Termites promote soil carbon and nitrogen depletion: Results from an in situ macrofauna exclusion experiment, Peru. Soil Biology and Biochemistry 77:109-111. DOI: 10.1016/j.soilbio.2014.05.033.

DeAngelis KM., Allgaier M., Chavarria Y., Fortney JL., Hugenholtz P., Simmons B., Sublette K., Silver WL., Hazen TC. 2011. Characterization of trapped lignin-degrading microbes in tropical forest soil. PLoS One 6:e19306. DOI: 10.1371/journal.pone.0019306.

Dietrich C., Brune A. 2016. The complete mitogenomes of six higher termite species reconstructed from metagenomic datasets ( Cornitermes sp., Cubitermes ugandensis, Microcerotermes parvus, Nasutitermes corniger, Neocapritermes taracua, and Termes hospes ). Mitochondrial DNA 27:3903-3904. DOI: 10.3109/19401736.2014.987257.

Dietrich C., Köhler T., Brune A. 2014. The cockroach origin of the termite gut microbiota: Patterns in bacterial community structure reflect major evolutionary events. Applied and Environmental Microbiology 80:2261-2269. DOI: 10.1128/AEM.04206-13.

Diouf M., Hervé V., Mora P., Robert A., Frechault S., Rouland-Lefèvre C., Miambi E. 2018a. Evidence from the gut microbiota of swarming alates of a vertical transmission of the bacterial symbionts in Nasutitermes arborum (Termitidae, Nasutitermitinae). Antonie van Leeuwenhoek 111:573-587. DOI: 10.1007/s10482-017-0978-4.

Diouf M., Miambi E., Mora P., Frechault S., Robert A., Rouland-Lefèvre C., Hervé V. $2018 b$. Variations in the relative abundance of Wolbachia in the gut of Nasutitermes arborum across life stages and castes. FEMS Microbiology Letters 365. DOI: 10.1093/femsle/fny046.

Donovan SE., Eggleton P., Bignell DE. 2001. Gut content analysis and a new feeding group classification of termites. Ecological Entomology 26:356-366. DOI: 10.1046/j.1365-

Peer] reviewing PDF | (2019:08:40743:1:1:NEW 19 Dec 2019) 
757

758

759

760

761

762

763

764

765

766

767

768

769

770

771

772

773

774

775

776

777

778

779

780

781

782

783

784

785

786

787

788

789

790

791

2311.2001.00342.x.

Friedrich MW., Schmitt-Wagner D., Lueders T., Brune A. 2001. Axial differences in community structure of Crenarchaeota and Euryarchaeota in the highly compartmentalized gut of the soil-feeding termite Cubitermes orthognathus. Applied and Environmental Microbiology 67:4880-4890. DOI: 10.1128/AEM.67.10.4880-4890.2001.

Fujita A., Miura T., Matsumoto T. 2008. Differences in cellulose digestive systems among castes in two termite lineages. Physiological Entomology 33:73-82. DOI: 10.1111/j.13653032.2007.00606.x.

Graber JR., Leadbetter JR., Breznak JA. 2004. Description of Treponema azotonutricium sp. nov. and Treponema primitia sp. nov., the first spirochetes isolated from termite guts. Applied and Environmental Microbiology 70:1315-1320. DOI: 10.1128/AEM.70.3.13151320.2004.

Grech-Mora I., Fardeau M-L., Patel BKC., Ollivier B., Rimbault A., Prensier G., Garcia J-L., Garnier-Sillam E. 1996. Isolation and characterization of Sporobacter termitidis gen. nov., sp. nov., from the digestive tract of the wood-feeding termite Nasutitermes lujae. International Journal of Systematic Bacteriology 46:512-518. DOI: 10.1099/00207713-46$2-512$.

Griffiths HM., Ashton LA., Evans TA., Parr CL., Eggleton P. 2019. Termites can decompose more than half of deadwood in tropical rainforest. Current Biology 29:R118-R119. DOI: 10.1016/j.cub.2019.01.012.

Guindon S., Dufayard JF., Lefort V., Anisimova M., Hordijk W., Gascuel O. 2010. New algorithms and methods to estimate maximum-likelihood phylogenies: Assessing the performance of PhyML 3.0. Systematic Biology 59:307-321. DOI: 10.1093/sysbio/syq010.

Gupta RS., Mahmood S., Adeolu M. 2013. A phylogenomic and molecular signature based approach for characterization of the phylum Spirochaetes and its major clades: proposal for a taxonomic revision of the phylum. Frontiers in microbiology 4:217. DOI: 10.3389/fmicb.2013.00217.

He S., Ivanova N., Kirton E., Allgaier M., Bergin C., Scheffrahn RH., Kyrpides NC., Warnecke F., Tringe SG., Hugenholtz P. 2013. Comparative metagenomic and metatranscriptomic analysis of hindgut paunch microbiota in wood- and dung-feeding higher termites. PloS one 8:e61126. DOI: 10.1371/journal.pone.0061126.

Herlemann DPR., Geissinger O., Ikeda-Ohtsubo W., Kunin V., Sun H., Lapidus A., Hugenholtz P., Brune A. 2009. Genomic analysis of "Elusimicrobium minutum," the first cultivated representative of the phylum "Elusimicrobia" (formerly termite group 1). Applied and Environmental Microbiology 75:2841-2849. DOI: 10.1128/AEM.02698-08. 
792 Hervé V., Brune A. 2017. The complete mitochondrial genomes of the higher termites

793 Labiotermes labralis and Embiratermes neotenicus (Termitidae: Syntermitinae).

794 Mitochondrial DNA Part B 2:109-110. DOI: 10.1080/23802359.2017.1289349.

795 Hervé V., Le Roux X., Uroz S., Gelhaye E., Frey-Klett P. 2014. Diversity and structure of

796 bacterial communities associated with Phanerochaete chrysosporium during wood decay.

797 Environmental Microbiology 16:2238-2252. DOI: 10.1111/1462-2920.12347.

798

799

800

801

802

803

804

805

806

807

808

809

810

811

812

813

814

815

816

817

818

819

820

821

822

823

824

825

826

Hongoh Y., Deevong P., Hattori S., Inoue T., Noda S., Noparatnaraporn N., Kudo T., Ohkuma M. 2006. Phylogenetic diversity, localization, and cell morphologies of members of the candidate phylum TG3 and a subphylum in the phylum Fibrobacteres, recently discovered bacterial groups dominant in termite guts. Applied and Environmental Microbiology 72:6780-8. DOI: 10.1128/AEM.00891-06.

Hongoh Y., Deevong P., Inoue T., Moriya S., Trakulnaleamsai S., Ohkuma M., Vongkaluang C., Noparatnaraporn N., Kudo T. 2005. Intra- and interspecific comparisons of bacterial diversity and community structure support coevolution of gut microbiota and termite host. Applied and Environmental Microbiology 71:6590-9. DOI: 10.1128/AEM.71.11.65906599.2005 .

Hongoh Y., Sharma VK., Prakash T., Noda S., Taylor TD., Kudo T., Sakaki Y., Toyoda A., Hattori M., Ohkuma M. 2008a. Complete genome of the uncultured Termite Group 1 bacteria in a single host protist cell. Proceedings of the National Academy of Sciences 105:5555-5560. DOI: 10.1073/pnas.0801389105.

Hongoh Y., Sharma VK., Prakash T., Noda S., Toh H., Taylor TD., Kudo T., Sakaki Y., Toyoda A., Hattori M., Ohkuma M. 2008b. Genome of an endosymbiont coupling N2 fixation to cellulolysis within protist cells in termite gut. Science (New York, N.Y.) 322:1108-9. DOI: $10.1126 /$ science. 1165578 .

Hua Z-S., Wang Y-L., Evans PN., Qu Y-N., Goh KM., Rao Y-Z., Qi Y-L., Li Y-X., Huang M-J., Jiao J-Y., Chen Y-T., Mao Y-P., Shu W-S., Hozzein W., Hedlund BP., Tyson GW., Zhang T., Li W-J. 2019. Insights into the ecological roles and evolution of methyl-coenzyme M reductase-containing hot spring Archaea. Nature Communications 10:4574. DOI: 10.1038/s41467-019-12574-y.

Kang DD., Li F., Kirton E., Thomas A., Egan R., An H., Wang Z. 2019. MetaBAT 2: an adaptive binning algorithm for robust and efficient genome reconstruction from metagenome assemblies. PeerJ 7:e7359. DOI: 10.7717/peerj.7359.

Katoh K., Standley DM. 2013. MAFFT multiple sequence alignment software version 7: improvements in performance and usability. Molecular Biology and Evolution 30:772-80. DOI: $10.1093 / \mathrm{molbev} / \mathrm{mst} 010$. 
827 Köhler T., Dietrich C., Scheffrahn RH., Brune A. 2012. High-resolution analysis of gut 828 environment and bacterial microbiota reveals functional compartmentation of the gut in

829

830

831

832

833

834

835

836

837

838

839

840

841

842

843

844

845

846

847

848

849

850

851

852

853

854

855

856

857

858

859

860 wood-feeding higher termites (Nasutitermes spp.). Applied and Environmental Microbiology 78:4691-701. DOI: 10.1128/AEM.00683-12.

Köhler T., Stingl U., Meuser K., Brune A. 2008. Novel lineages of Planctomycetes densely colonize the alkaline gut of soil-feeding termites (Cubitermes spp.). Environmental Microbiology 10:1260-1270. DOI: 10.1111/j.1462-2920.2007.01540.x.

Krishna K., Grimaldi DA., Krishna V., Engel MS. 2013. Treatise on the Isoptera of the World. Bulletin of the American Museum of Natural History 377:2433-2705. DOI: 10.1206/377.7.

Kuhnigk T., Branke J., Krekeler D., Cypionka H., König H. 1996. A feasible role of sulfatereducing bacteria in the termite gut. Systematic and Applied Microbiology 19:139-149. DOI: $10.1016 / \mathrm{S} 0723-2020(96) 80039-7$.

Lampert N., Mikaelyan A., Brune A. 2019. Diet is not the primary driver of bacterial community structure in the gut of litter-feeding cockroaches. BMC Microbiology 19:238. DOI: 10.1186/s12866-019-1601-9.

Lazar CS., Baker BJ., Seitz K., Hyde AS., Dick GJ., Hinrichs K-U., Teske AP. 2016. Genomic evidence for distinct carbon substrate preferences and ecological niches of Bathyarchaeota in estuarine sediments. Environmental Microbiology 18:1200-1211. DOI: 10.1111/14622920.13142 .

Leadbetter JR., Schmidt TM., Graber JR., Breznak JA. 1999. Acetogenesis from H2 plus CO2 by spirochetes from termite guts. Science (New York, N.Y.) 283:686-9.

Lefort V., Longueville J-E., Gascuel O. 2017. SMS: Smart Model Selection in PhyML. Molecular Biology and Evolution 6:461-464. DOI: 10.1093/molbev/msx 149 .

Letunic I., Bork P. 2019. Interactive Tree Of Life (iTOL) v4: recent updates and new developments. Nucleic Acids Research. DOI: 10.1093/nar/gkz239.

Li H., Durbin R. 2009. Fast and accurate short read alignment with Burrows-Wheeler transform. Bioinformatics (Oxford, England) 25:1754-60. DOI: 10.1093/bioinformatics/btp324.

Li H., Handsaker B., Wysoker A., Fennell T., Ruan J., Homer N., Marth G., Abecasis G., Durbin R. 2009. The Sequence Alignment/Map format and SAMtools. Bioinformatics 25:20782079. DOI: $10.1093 /$ bioinformatics/btp352.

Lilburn., Schmidt., Breznak. 1999. Phylogenetic diversity of termite gut spirochaetes. Environmental Microbiology 1:331-345. DOI: 10.1046/j.1462-2920.1999.00043.x.

Liu G., Cornwell WK., Cao K., Hu Y., Van Logtestijn RSP., Yang S., Xie X., Zhang Y., Ye D., Pan X., Ye X., Huang Z., Dong M., Cornelissen JHC. 2015. Termites amplify the effects of 
861 wood traits on decomposition rates among multiple bamboo and dicot woody species. Journal of Ecology 103:1214-1223. DOI: 10.1111/1365-2745.12427.

863 Lombard V., Golaconda Ramulu H., Drula E., Coutinho PM., Henrissat B. 2014. The 864 carbohydrate-active enzymes database (CAZy) in 2013. Nucleic Acids Research 42:D490865 D495. DOI: 10.1093/nar/gkt1178.

866 Ludwig W., Strunk O., Westram R., Richter L., Meier H., Yadhukumar., Buchner A., Lai T.,

867 Steppi S., Jobb G., Förster W., Brettske I., Gerber S., Ginhart AW., Gross O., Grumann S., 868 Hermann S., Jost R., König A., Liss T., Lüssmann R., May M., Nonhoff B., Reichel B., 869

Marynowska M., Goux X., Sillam-Dussès D., Rouland-Lefèvre C., Roisin Y., Delfosse P., Calusinska M. 2017. Optimization of a metatranscriptomic approach to study the lignocellulolytic potential of the higher termite gut microbiome. BMC Genomics 18:681. DOI: $10.1186 / \mathrm{s} 12864-017-4076-9$.

Mikaelyan A., Dietrich C., Köhler T., Poulsen M., Sillam-Dussès D., Brune A. 2015a. Diet is the primary determinant of bacterial community structure in the guts of higher termites. Molecular Ecology 24:5284-5295. DOI: 10.1111/mec.13376.

Mikaelyan A., Köhler T., Lampert N., Rohland J., Boga H., Meuser K., Brune A. 2015b. Classifying the bacterial gut microbiota of termites and cockroaches: A curated phylogenetic reference database (DictDb). Systematic and Applied Microbiology 38:472482. DOI: 10.1016/j.syapm.2015.07.004.

Mikaelyan A., Meuser K., Brune A. 2017. Microenvironmental heterogeneity of gut compartments drives bacterial community structure in wood- and humus-feeding higher termites. FEMS Microbiology Ecology 93:fiw210. DOI: 10.1093/femsec/fiw210.

Mikaelyan A., Strassert JFH., Tokuda G., Brune A. 2014. The fibre-associated cellulolytic bacterial community in the hindgut of wood-feeding higher termites (Nasutitermes spp.). Environmental Microbiology 16:2711-2722. DOI: 10.1111/1462-2920.12425. 
896

897

898

899

900

901

902

903

904

905

906

907

908

909

910

911

912

913

914

915

916

917

918

919

920

921

922

923

924

925

926

927

928

929

of the humus- and litter-feeding termite Procornitermes araujoi (Syntermitinae). Current Microbiology:1-10. DOI: 10.1007/s00284-018-1567-0.

Neumann AP., McCormick CA., Suen G. 2017. Fibrobacter communities in the gastrointestinal tracts of diverse hindgut-fermenting herbivores are distinct from those of the rumen. Environmental Microbiology 19:3768-3783. DOI: 10.1111/1462-2920.13878.

Neumann AP., Suen G. 2018. The phylogenomic diversity of herbivore-associated Fibrobacter spp. is correlated to lignocellulose-degrading potential. mSphere 3:e00593-18. DOI: 10.1128/mSphere.00593-18.

Odelson DA., Breznak JA. 1983. Volatile fatty acid production by the hindgut microbiota of xylophagous termites. Applied and Environmental Microbiology 45:1602-13.

Ohkuma M., Iida T., Kudo T. 1999. Phylogenetic relationships of symbiotic spirochetes in the gut of diverse termites. FEMS Microbiology Letters 181:123-129.

Ohkuma M., Noda S., Hattori S., Iida T., Yuki M., Starns D., Inoue J., Darby AC., Hongoh Y. 2015. Acetogenesis from $\mathrm{H} 2$ plus $\mathrm{CO} 2$ and nitrogen fixation by an endosymbiotic spirochete of a termite-gut cellulolytic protist. Proceedings of the National Academy of Sciences 112:10224-10230. DOI: 10.1073/pnas.1423979112.

Ohkuma M., Noda S., Kudo T. 1999. Phylogenetic diversity of nitrogen fixation genes in the symbiotic microbial community in the gut of diverse termites. Applied and Environmental Microbiology 65:4926-34.

Ottesen EA., Leadbetter JR. 2011. Formyltetrahydrofolate synthetase gene diversity in the guts of higher termites with different diets and lifestyles. Applied and Environmental Microbiology 77:3461-3467. DOI: 10.1128/AEM.02657-10.

Parks DH., Chuvochina M., Waite DW., Rinke C., Skarshewski A., Chaumeil P-A., Hugenholtz P. 2018. A standardized bacterial taxonomy based on genome phylogeny substantially revises the tree of life. Nature Biotechnology. DOI: 10.1038/nbt.4229.

Parks DH., Imelfort M., Skennerton CT., Hugenholtz P., Tyson GW. 2015. CheckM: assessing the quality of microbial genomes recovered from isolates, single cells, and metagenomes. Genome research 25:1043-55. DOI: 10.1101/gr.186072.114.

Parks DH., Rinke C., Chuvochina M., Chaumeil P-A., Woodcroft BJ., Evans PN., Hugenholtz P., Tyson GW. 2017. Recovery of nearly 8,000 metagenome-assembled genomes substantially expands the tree of life. Nature Microbiology 2:1533-1542. DOI: 10.1038/s41564-017-0012-7.

Paster BJ., Dewhirst FE., Cooke SM., Fussing V., Poulsen LK., Breznak JA. 1996. Phylogeny of not-yet-cultured spirochetes from termite guts. Applied and Environmental Microbiology 
930

931

932

933

934

935

936

937

938

939

940

941

942

943

944

945

946

947

948

949

950

951

952

953

954

955

956

957

958

959

960

961

962

963

62:347-52.

Prosser JI. 2015. Dispersing misconceptions and identifying opportunities for the use of "omics" in soil microbial ecology. Nature Reviews Microbiology 13:439-446. DOI: 10.1038/nrmicro3468.

Pruesse E., Peplies J., Glöckner FO. 2012. SINA: accurate high-throughput multiple sequence alignment of ribosomal RNA genes. Bioinformatics 28:1823-1829. DOI: 10.1093/bioinformatics/bts252.

Qiu YL., Kuang XZ., Shi XS., Yuan XZ., Guo RB. 2014. Paludibacter jiangxiensis sp. nov., a strictly anaerobic, propionate-producing bacterium isolated from rice paddy field. Archives of Microbiology 196:149-155. DOI: 10.1007/s00203-013-0951-1.

Quast C., Pruesse E., Yilmaz P., Gerken J., Schweer T., Yarza P., Peplies J., Glöckner FO. 2013. The SILVA ribosomal RNA gene database project: improved data processing and webbased tools. Nucleic acids research 41:D590-6. DOI: 10.1093/nar/gks1219.

R Development Core Team. 2019. R: A language and environment for statistical computing.

Le Roes-Hill M., Rohland J., Burton S. 2011. Actinobacteria isolated from termite guts as a source of novel oxidative enzymes. Antonie van Leeuwenhoek 100:589-605. DOI: 10.1007/s10482-011-9614-x.

Rossmassler K., Dietrich C., Thompson C., Mikaelyan A., Nonoh JO., Scheffrahn RH., SillamDussès D., Brune A. 2015. Metagenomic analysis of the microbiota in the highly compartmented hindguts of six wood- or soil-feeding higher termites. Microbiome 3:56. DOI: $10.1186 / \mathrm{s} 40168-015-0118-1$.

Sabree ZL., Moran NA. 2014. Host-specific assemblages typify gut microbial communities of related insect species. SpringerPlus 3:138. DOI: 10.1186/2193-1801-3-138.

Schloss PD., Girard RA., Martin T., Edwards J., Thrash JC. 2016. Status of the Archaeal and Bacterial census: An update. mBio 7:e00201-16. DOI: 10.1128/mBio.00201-16.

Schmidt O., Horn MA., Kolb S., Drake HL. 2015. Temperature impacts differentially on the methanogenic food web of cellulose-supplemented peatland soil. Environmental Microbiology 17:720-734. DOI: 10.1111/1462-2920.12507.

Schulz F., Eloe-Fadrosh EA., Bowers RM., Jarett J., Nielsen T., Ivanova NN., Kyrpides NC., Woyke T. 2017. Towards a balanced view of the bacterial tree of life. Microbiome 5:140. DOI: $10.1186 / \mathrm{s} 40168-017-0360-9$.

Sczyrba A., Hofmann P., Belmann P., Koslicki D., Janssen S., Dröge J., Gregor I., Majda S., Fiedler J., Dahms E., Bremges A., Fritz A., Garrido-Oter R., Jørgensen TS., Shapiro N., Blood PD., Gurevich A., Bai Y., Turaev D., DeMaere MZ., Chikhi R., Nagarajan N., 
964

965

966

967

968

969

970

971

972

973

974

975

976

977

978

979

980

981

982

983

984

985

986

987

988

989

990

991

992

993

994

995

996

997

998

999

Quince C., Meyer F., Balvočiūtè M., Hansen LH., Sørensen SJ., Chia BKH., Denis B., Froula JL., Wang Z., Egan R., Don Kang D., Cook JJ., Deltel C., Beckstette M., Lemaitre C., Peterlongo P., Rizk G., Lavenier D., Wu Y-W., Singer SW., Jain C., Strous M., Klingenberg H., Meinicke P., Barton MD., Lingner T., Lin H-H., Liao Y-C., Silva GGZ., Cuevas DA., Edwards RA., Saha S., Piro VC., Renard BY., Pop M., Klenk H-P., Göker M., Kyrpides NC., Woyke T., Vorholt JA., Schulze-Lefert P., Rubin EM., Darling AE., Rattei T., McHardy AC. 2017. Critical Assessment of Metagenome Interpretation - a benchmark of metagenomics software. Nature Methods 14:1063-1071. DOI: 10.1038/nmeth.4458.

Shi Y., Huang Z., Han S., Fan S., Yang H. 2015. Phylogenetic diversity of Archaea in the intestinal tract of termites from different lineages. Journal of Basic Microbiology 55:10211028. DOI: $10.1002 /$ jobm.201400678.

Söllinger A., Tveit AT., Poulsen M., Noel SJ., Bengtsson M., Bernhardt J., Frydendahl Hellwing AL., Lund P., Riedel K., Schleper C., Højberg O., Urich T. 2018. Holistic assessment of rumen microbiome dynamics through quantitative metatranscriptomics reveals multifunctional redundancy during key steps of anaerobic feed degradation. mSystems 3:e00038-18. DOI: 10.1128/mSystems.00038-18.

Sorokin DY., Rakitin AL., Gumerov VM., Beletsky A V., Sinninghe Damsté JS., Mardanov A V., Ravin N V. 2016. Phenotypic and genomic properties of Chitinispirillum alkaliphilum gen. nov., sp. nov., a haloalkaliphilic anaerobic chitinolytic bacterium representing a novel class in the phylum Fibrobacteres. Frontiers in Microbiology 7:407. DOI: 10.3389/fmicb.2016.00407.

Spring S., Bunk B., Spröer C., Schumann P., Rohde M., Tindall BJ., Klenk H-P. 2016. Characterization of the first cultured representative of Verrucomicrobia subdivision 5 indicates the proposal of a novel phylum. The ISME Journal 10:2801-2816. DOI: 10.1038/ismej.2016.84.

Stewart RD., Auffret MD., Warr A., Wiser AH., Press MO., Langford KW., Liachko I., Snelling TJ., Dewhurst RJ., Walker AW., Roehe R., Watson M. 2018. Assembly of 913 microbial genomes from metagenomic sequencing of the cow rumen. Nature Communications 9:870. DOI: $10.1038 / \mathrm{s} 41467-018-03317-6$.

Sujada N., Sungthong R., Lumyong S. 2014. Termite nests as an abundant source of cultivable Actinobacteria for biotechnological purposes. Microbes and Environments 29:211-219. DOI: $10.1264 /$ jsme2.ME13183.

Svartström O., Alneberg J., Terrapon N., Lombard V., de Bruijn I., Malmsten J., Dalin A-M., EL Muller E., Shah P., Wilmes P., Henrissat B., Aspeborg H., Andersson AF. 2017. Ninetynine de novo assembled genomes from the moose (Alces alces) rumen microbiome provide new insights into microbial plant biomass degradation. The ISME Journal 11:2538-2551.

Peer) reviewing PDF | (2019:08:40743:1:1:NEW 19 Dec 2019) 
DOI: $10.1038 /$ ismej.2017.108.

1001 Tokuda G., Lo N., Watanabe H., Arakawa G., Matsumoto T., Noda H. 2004. Major alteration of the expression site of endogenous cellulases in members of an apical termite lineage. Molecular Ecology 13:3219-3228. DOI: 10.1111/j.1365-294X.2004.02276.x.

1004 Tokuda G., Mikaelyan A., Fukui C., Matsuura Y., Watanabe H., Fujishima M., Brune A. 2018. Fiber-associated spirochetes are major agents of hemicellulose degradation in the hindgut of wood-feeding higher termites. Proceedings of the National Academy of Sciences 115:E11996-E12004. DOI: 10.1073/pnas.1810550115.

Tokuda G., Tsuboi Y., Kihara K., Saitou S., Moriya S., Lo N., Kikuchi J. 2014. Metabolomic profiling of 13C-labelled cellulose digestion in a lower termite: insights into gut symbiont function. Proceedings. Biological sciences / The Royal Society 281:20140990. DOI: 10.1098/rspb.2014.0990.

1013

Ueki A., Akasaka H., Suzuki D., Ueki K. 2006. Paludibacter propionicigenes gen. nov., sp. nov., a novel strictly anaerobic, Gram-negative, propionate-producing bacterium isolated from plant residue in irrigated rice-field soil in Japan. International Journal of Systematic and Evolutionary Microbiology 56:39-44. DOI: 10.1099/ijs.0.63896-0.

Utami YD., Kuwahara H., Igai K., Murakami T., Sugaya K., Morikawa T., Nagura Y., Yuki M.,

Utami YD., Kuwahara H., Murakami T., Morikawa T., Sugaya K., Kihara K., Yuki M., Lo N., Deevong P., Hasin S., Boonriam W., Inoue T., Yamada A., Ohkuma M., Hongoh Y. 2018. Phylogenetic diversity and single-cell genome analysis of "Melainabacteria", a nonphotosynthetic cyanobacterial group, in the termite gut. Microbes and Environments 33:5057. DOI: 10.1264/jsme2.ME17137. Diversity and resilience of the wood-feeding higher termite Mironasutitermes

Wertz JT., Kim E., Breznak JA., Schmidt TM., Rodrigues JLM. 2012. Genomic and physiological characterization of the Verrucomicrobia isolate Diplosphaera colitermitum gen. nov., sp. nov., reveals microaerophily and nitrogen fixation genes. Applied and

1034 Wickham H. 2016. ggplot2: Elegant graphics for data analysis. Springer-Verlag New York. 
DOI: 10.1007/978-3-319-24277-4.

1036

1037

1038

1039

1040

1041

1042

1043

1044

1045

1046

1047

1048

1049

1050

1051

1052

1053

1054

Woyke T., Doud DFR., Schulz F. 2017. The trajectory of microbial single-cell sequencing. Nature Methods 14:1045-1054. DOI: 10.1038/nmeth.4469.

Yamada A., Inoue T., Wiwatwitaya D., Ohkuma M., Kudo T., Abe T., Sugimoto A. 2005. Carbon mineralization by termites in tropical forests, with emphasis on fungus combs. Ecological Research 20:453-460.

Yuki M., Kuwahara H., Shintani M., Izawa K., Sato T., Starns D., Hongoh Y., Ohkuma M. 2015. Dominant ectosymbiotic bacteria of cellulolytic protists in the termite gut also have the potential to digest lignocellulose. Environmental Microbiology 17:4942-4953. DOI: 10.1111/1462-2920.12945.

Yuki M., Sakamoto M., Nishimura Y., Ohkuma M. 2018. Lactococcus reticulitermitis sp. nov., isolated from the gut of the subterranean termite Reticulitermes speratus. International Journal of Systematic and Evolutionary Microbiology 68:596-601. DOI: 10.1099/ijsem.0.002549.

Zheng H., Brune A. 2015. Complete genome sequence of Endomicrobium proavitum, a freeliving relative of the intracellular symbionts of termite gut flagellates (phylum Elusimicrobia). Genome Announcements 3:e0679-15. DOI: 10.1128/genomeA.00679-15.

Zhou Z., Pan J., Wang F., Gu J-D., Li M. 2018. Bathyarchaeota: globally distributed metabolic generalists in anoxic environments. FEMS Microbiology Reviews. DOI: 10.1093/femsre/fuy023. 
Figure 1

Relationship between the number of MAGs recovered and the number of assembled reads in the respective metagenomes.

The linear regression line and the Pearson correlation coefficient $(r)$ are shown for the entire dataset.

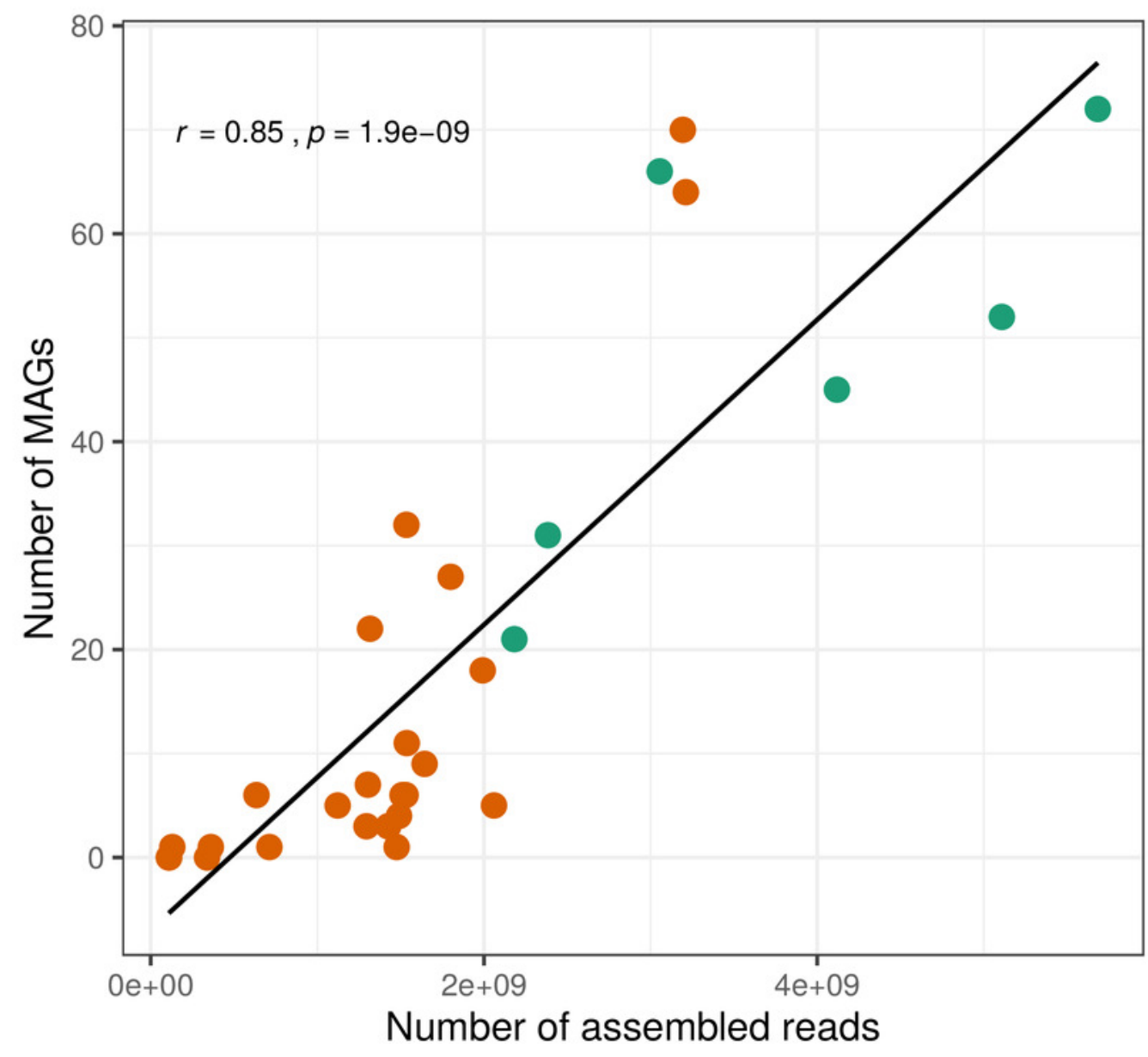

Dataset

Current

Rossmassler 2015 


\section{Figure 2}

Distribution of the 589 MAGs among bacterial and archaeal phyla.

This maximum-likelihood tree was inferred from a concatenated alignment (amino acids) of 43 protein-coding genes $(6,801$ positions) using the $L G+G+I$ model of evolution.

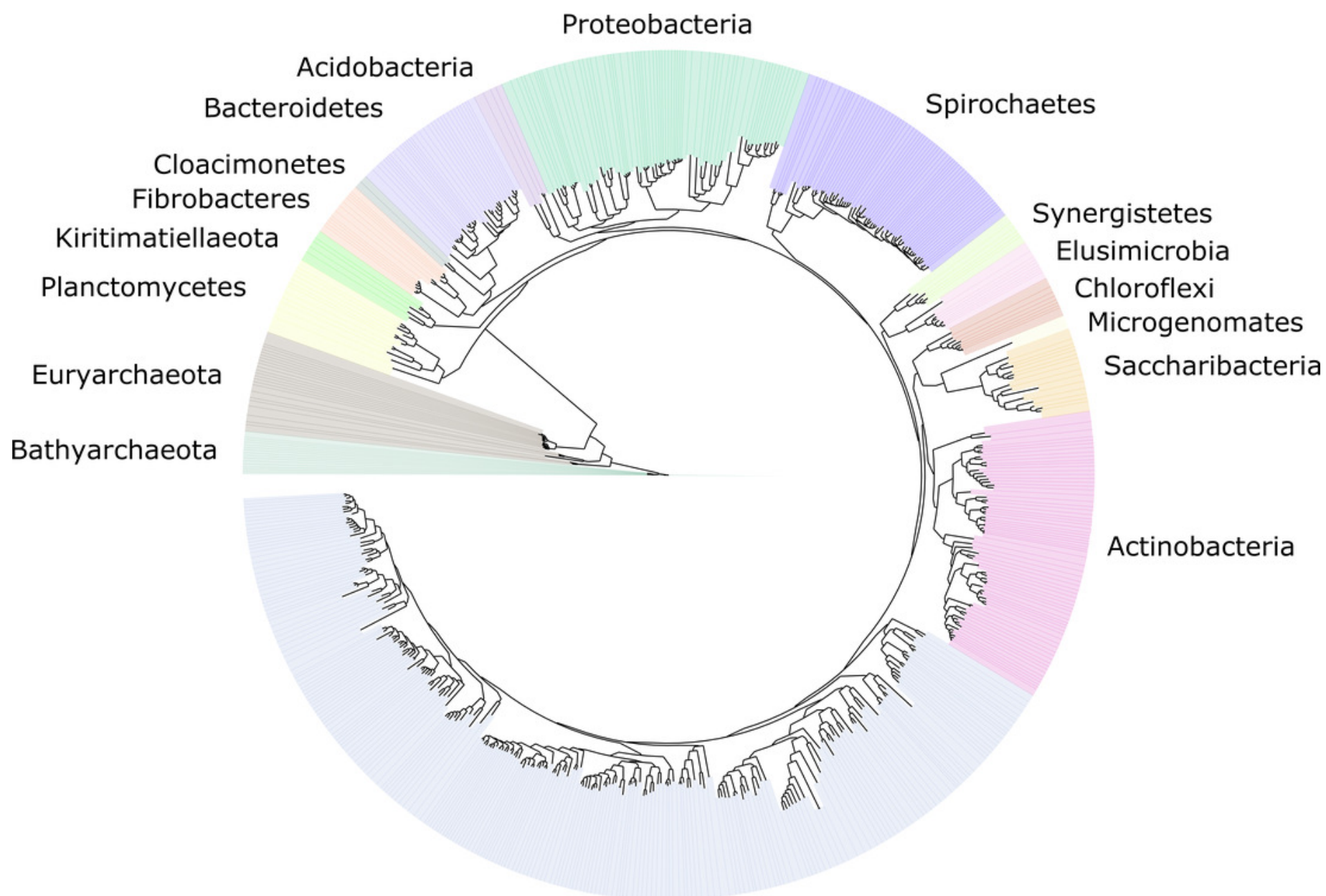

Firmicutes 
Figure 3

Phylum-level representation of MAGs among the bacterial gut microbiota of higher termites.

The average abundance of the corresponding lineages in 16S rRNA amplicon libraries of higher termites from different diet groups is shown for comparison. Core lineages represented in at least $80 \%$ of these samples are marked. For an interactive spreadsheet resolving each lineage to genus level, see Supplementary Information (Table S4).

\begin{tabular}{|c|c|c|c|c|c|c|c|c|c|c|}
\hline \multirow[t]{3}{*}{ Phylum } & \multicolumn{3}{|c|}{ MAGs } & \multicolumn{7}{|c|}{ 16S rRNA amplicon libraries } \\
\hline & \multirow{2}{*}{$\begin{array}{l}\text { Total } \\
\text { number }\end{array}$} & with & \multirow{2}{*}{$\begin{array}{l}\text { Rel. abd. } \\
\text { (\%) }\end{array}$} & \multirow{2}{*}{ ๑ัँ } & \multirow[b]{2}{*}{ All } & \multicolumn{3}{|c|}{ Average abundance (\%) } & \multirow[b]{2}{*}{ Soil } & \multirow[b]{2}{*}{ Fungus } \\
\hline & & $16 S$ gene & & & & Wood & Litter & Humus & & \\
\hline Acidobacteria & 4 & 2 & 0.4 & - & 0.5 & 0.7 & 0.4 & 0.8 & 0.4 & 0.2 \\
\hline Actinobacteria & 71 & 22 & 3.0 & - & 3.1 & 2.0 & 1.8 & 2.1 & 5.2 & 3.9 \\
\hline Bacteroidetes & 33 & 5 & 2.7 & - & 17.5 & 7.6 & 22.1 & 18.5 & 12.1 & 28.7 \\
\hline Chloroflexi & 8 & 5 & 0.1 & & 0.2 & 0.0 & 0.1 & 0.3 & 0.8 & 0.0 \\
\hline Cloacimonetes & 2 & 2 & 0.2 & & 0.4 & 0.0 & 0.2 & 1.6 & 0.2 & 0.0 \\
\hline Deferribacteres & - & - & & & 0.3 & 0.0 & 0.0 & 0.1 & 0.1 & 1.1 \\
\hline Elusimicrobia & 9 & 2 & 0.4 & - & 0.8 & 0.1 & 1.4 & 1.9 & 0.3 & 0.5 \\
\hline Epsilonproteobacteria & - & - & & & 1.5 & 0.3 & 0.7 & 0.0 & 0.2 & 6.1 \\
\hline Fibrobacteres & 13 & 2 & 6.6 & - & 3.2 & 8.5 & 6.8 & 0.6 & 0.1 & 1.1 \\
\hline Firmicutes & 237 & 73 & 9.2 & - & 33.5 & 13.1 & 26.0 & 41.0 & 56.2 & 28.7 \\
\hline Fusobacteria & - & - & & & 0.2 & 0.1 & 0.0 & 0.0 & 0.1 & 0.7 \\
\hline Kiritimatiellaeota & 5 & 3 & 0.3 & & 0.3 & 0.1 & 0.2 & 0.3 & 0.9 & 0.1 \\
\hline Microgenomates & 1 & 1 & 0.6 & - & 0.6 & 0.1 & 0.1 & 0.2 & 0.4 & 2.3 \\
\hline Planctomycetes & 12 & 8 & 0.7 & & 1.0 & 0.0 & 0.1 & 0.0 & 0.6 & 3.7 \\
\hline Proteobacteria & 67 & 23 & 9.8 & - & 7.8 & 3.5 & 8.0 & 7.7 & 9.4 & 10.6 \\
\hline Saccharibacteria & 15 & 12 & 0.1 & - & 2.2 & 0.4 & 3.1 & 1.9 & 0.8 & 5.2 \\
\hline Spirochaetes & 68 & 10 & 3.0 & - & 24.5 & 62.1 & 27.5 & 21.1 & 8.1 & 4.7 \\
\hline Synergistetes & 6 & 1 & 0.3 & - & 1.5 & 0.8 & 0.5 & 1.2 & 3.2 & 1.5 \\
\hline Verrucomicrobia & - & - & & - & 0.4 & 0.1 & 0.3 & 0.2 & 0.6 & 0.6 \\
\hline Bacteria & 551 & 170 & 36.7 & & 100 & 100 & 100 & 100 & 100 & 100 \\
\hline Bathyarchaeota & 15 & 10 & 1.2 & & & & & & & \\
\hline Euryarchaeota & 23 & 6 & 0.5 & & & & & & & \\
\hline Archaea & 38 & 16 & 1.7 & & & & & & & \\
\hline Total & 589 & 187 & 38.4 & & & & & & & \\
\hline
\end{tabular}


Figure 4

Relative abundance of the MAGs from different phyla among the respective metagenomes.

Circle size indicates the relative abundance of the MAGs among the respective metagenome sample; color indicates host diet. To estimate the relative abundance of each MAG, the total number of reads mapped to a MAG was divided by the total number of reads in the corresponding metagenome sample.

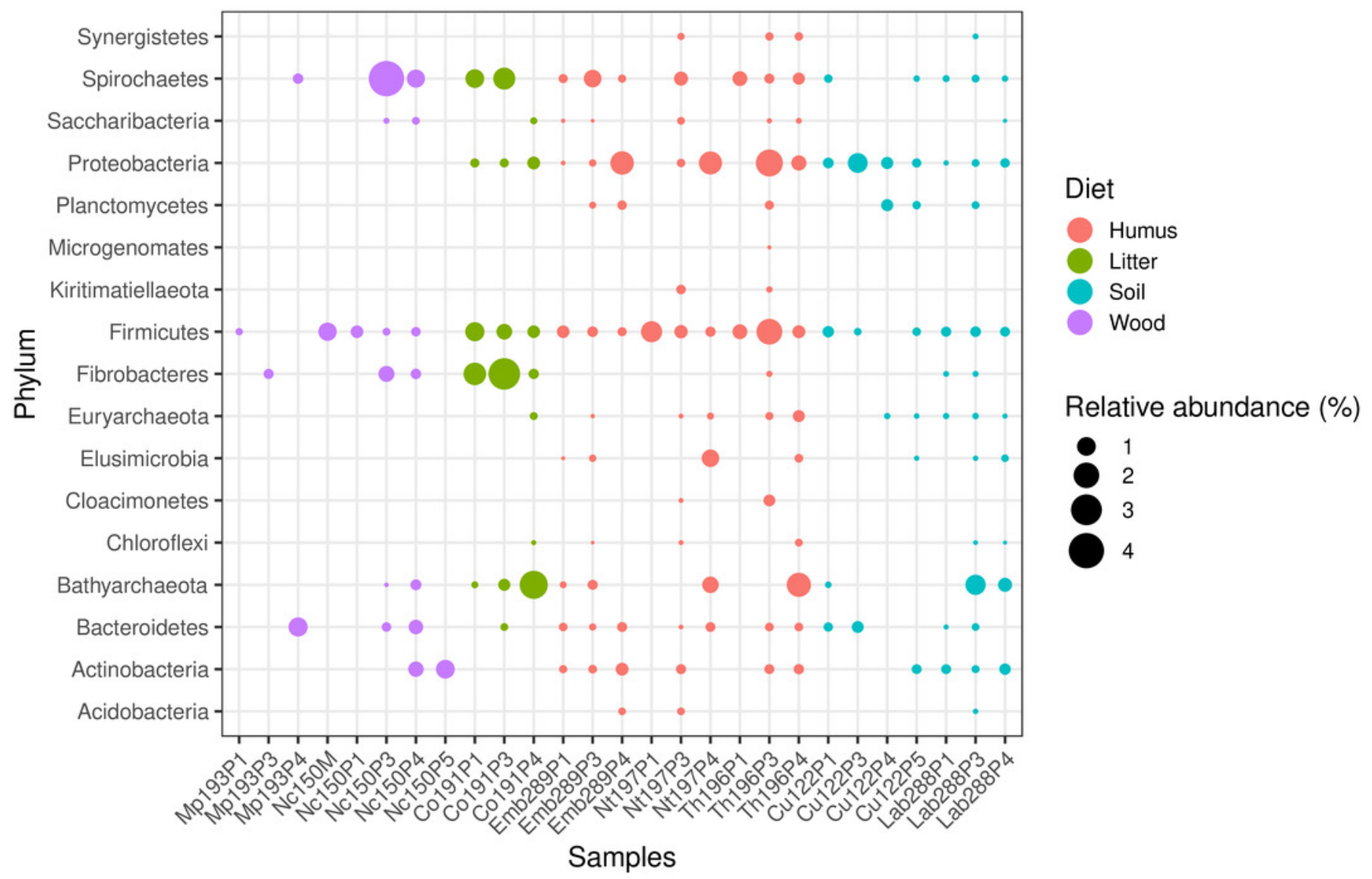




\section{Figure 5}

Phylogenomic tree of the archaeal domain.

This maximum-likelihood tree was inferred from a concatenated alignment of 43 proteins $(6,682$ positions) using the $L G+G+I+F$ model of amino-acid evolution. Branch supports were calculated using a Chi2-based parametric approximate likelihood-ratio test. Names in bold included MAGs recovered in the present study. Clusters shaded in brown consist exclusively of MAGs from termite guts and clusters shaded in gray contain genomes from termite guts. The Asgard group was used as outgroup.

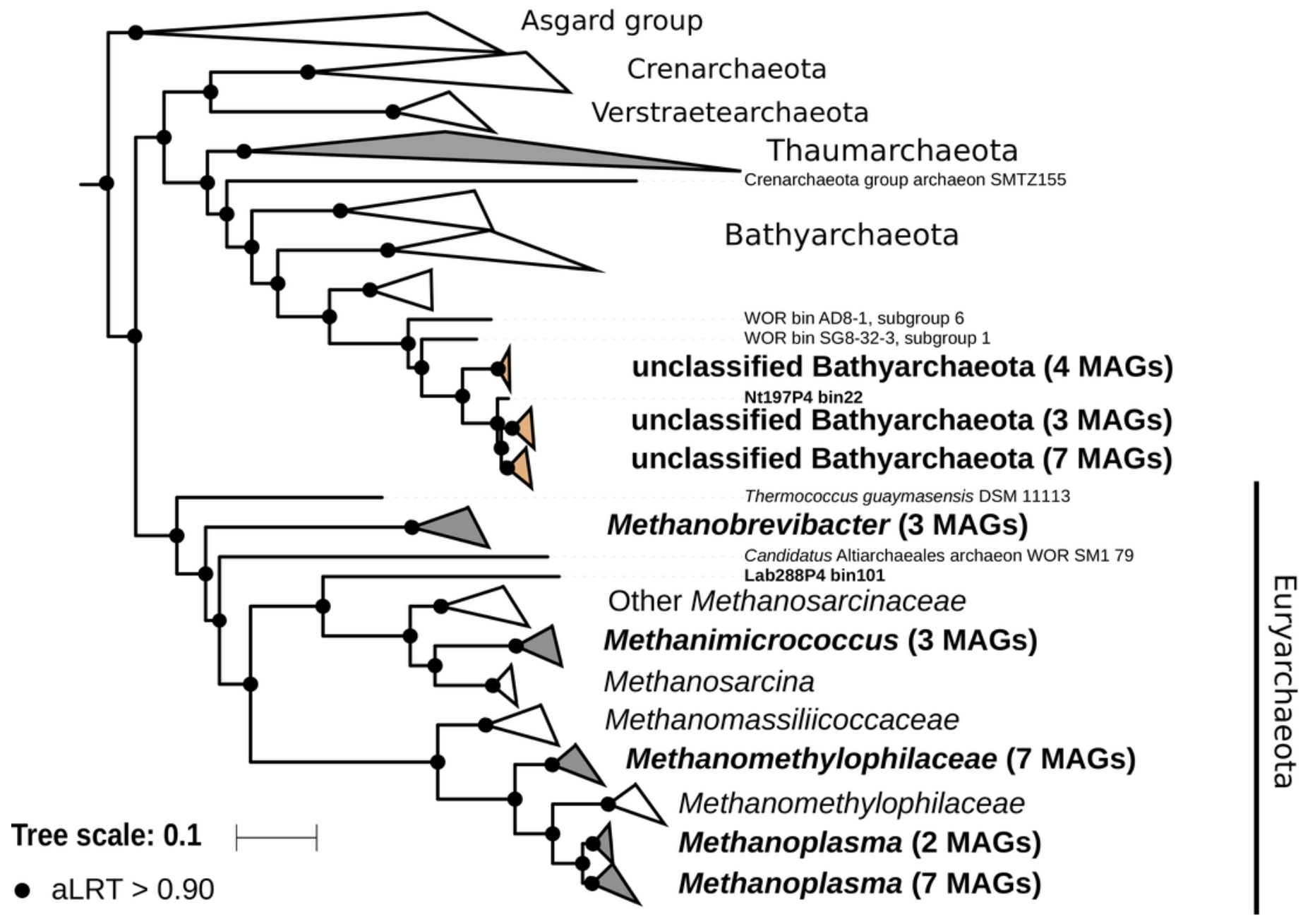




\section{Figure 6}

Phylogenomic tree of the Spirochaetes phylum.

This maximum-likelihood tree was inferred from a concatenated alignment of 43 proteins $(6,741$ positions) using the $L G+G+I+F$ model of amino-acid evolution. Branch supports were calculated using a Chi2-based parametric approximate likelihood-ratio test. Names in bold included MAGs recovered in the present study. Clusters shaded in brown consist exclusively of genomes from termite guts and clusters shaded in gray contain genomes from termite guts. Elusimicrobia and Cyanobacteria were used as outgroup. 


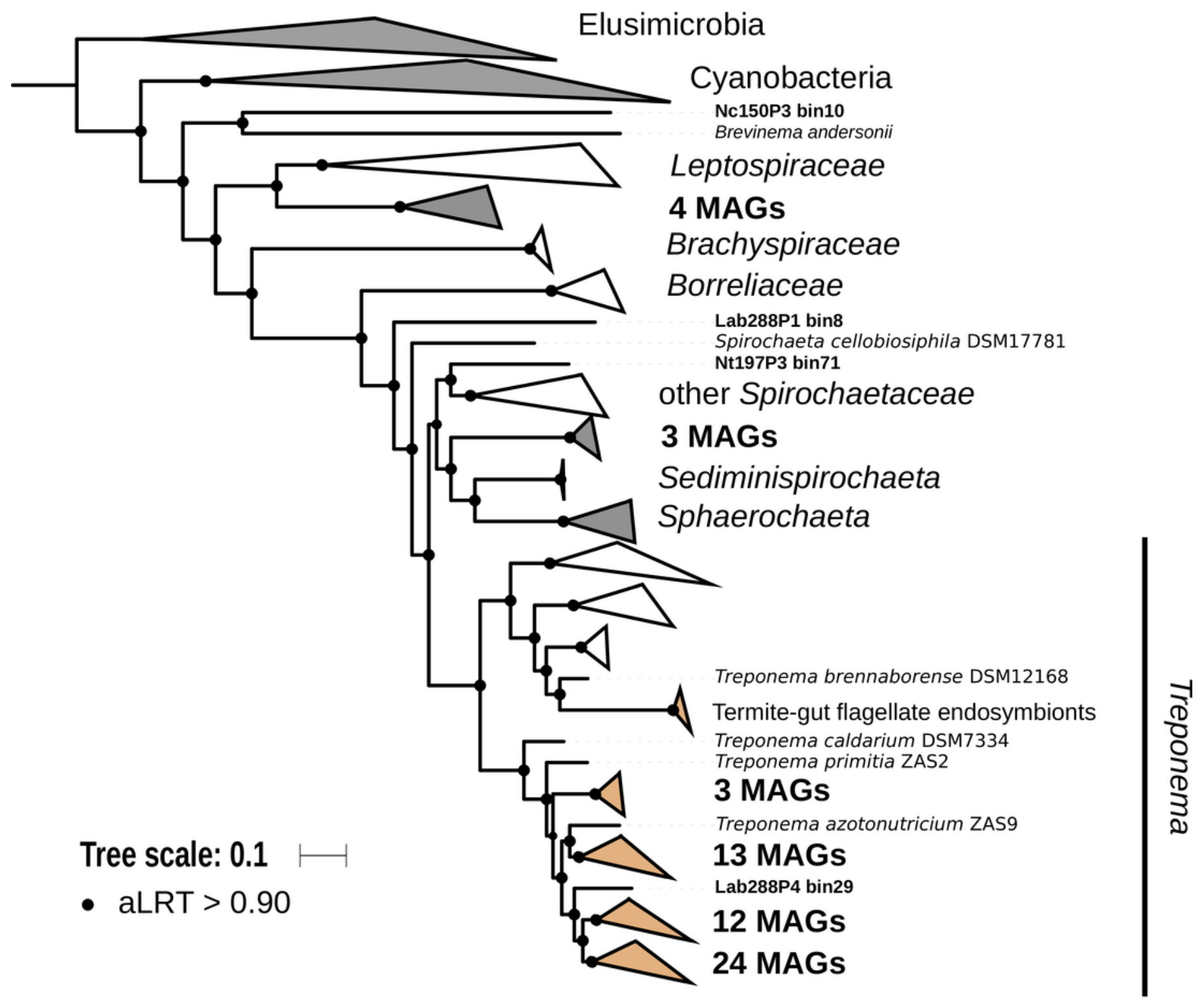




\section{Figure 7}

Phylogenomic tree of the Fibrobacteres phylum.

This maximum-likelihood tree was inferred from a concatenated alignment of 43 proteins (6,516 positions) using the $L G+G+I+F$ model of amino-acid evolution. Branch supports were calculated using a Chi2-based parametric approximate likelihood-ratio test. Names in bold included MAGs recovered in the present study. Clusters shaded in brown consist exclusively of genomes from termite guts and clusters shaded in gray contain genomes from termite guts. Bacteroidetes were used as outgroup.

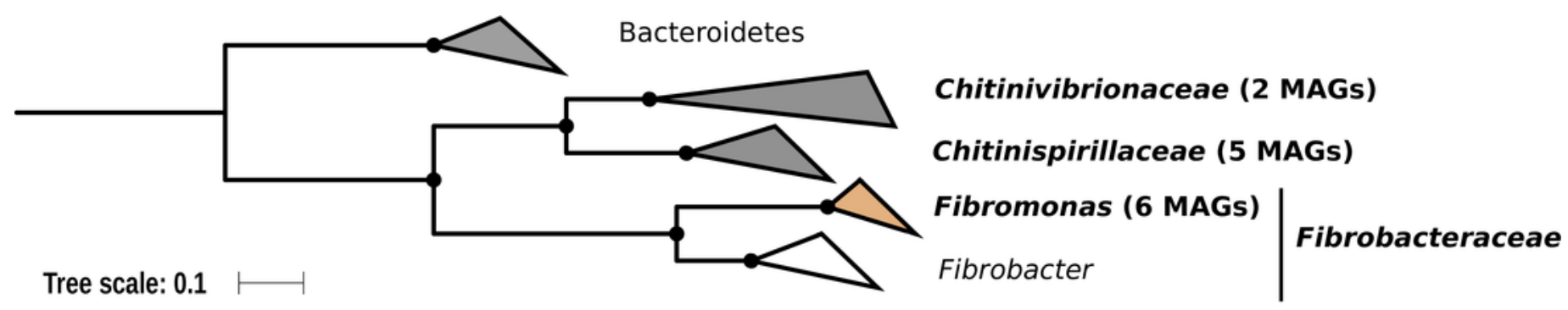

- aLRT $>0.95$ 


\section{Table $\mathbf{1}$ (on next page)}

Recovery of metagenome-assembled genomes (MAGs) from the 30 termite gut metagenomes analyzed in this study.

The host termite, its mitochondrial genome accession number, dietary preference, and the originating gut compartments are indicated. $C$ crop (foregut), $M$ midgut, P1-P5 proctodeal compartments (hindgut). The sample codes used for the metagenomes are the combination of host ID and gut compartment. 
1 Table 1. Recovery of metagenome-assembled genomes (MAGs) from the 30 termite gut 2 metagenomes analyzed in this study. The host termite, its mitochondrial genome accession 3 number, dietary preference, and the originating gut compartments are indicated. $C$ crop (foregut), $4 \quad M$ midgut, P1-P5 proctodeal compartments (hindgut). The sample codes used for the 5 metagenomes are the combination of host ID and gut compartment.

6

7

\begin{tabular}{|c|c|c|c|c|c|c|c|c|c|c|}
\hline Termite species & ID & Mitogenome & Diet & \multicolumn{4}{c|}{ Number of MAGs } \\
\hline & & & & C & M & P1 & P3 & P4 & P5 & Total \\
\hline Microcerotermes parvus & Mp193 & KP091690 & Wood & $-{ }^{\text {a }}$ & - & 1 & 1 & 4 & - & 6 \\
\hline Nasutitermes corniger & Nc150 & KP091691 & Wood & 0 & 1 & 3 & 6 & 9 & 1 & 20 \\
\hline Cornitermes sp. & Co191 & KP091688 & Litter & - & - & 32 & 22 & 7 & - & 61 \\
\hline Neocapritermes taracua & Nt197 & KP091692 & Humus & - & - & 6 & 70 & 11 & - & 87 \\
\hline Termes hospes & Th196 & KP091693 & Humus & - & - & 6 & 64 & 27 & - & 97 \\
\hline Embiratermes neotenicus & Emb289 & KY436202 & Humus & - & - & 45 & 52 & 21 & - & 118 \\
\hline Labiotermes labralis & Lab288 & KY436201 & Soil & - & - & 66 & 72 & 31 & - & 169 \\
\hline Cubitermes ugandensis & Cu122 & KP091689 & Soil & 0 & 0 & 5 & 5 & 3 & 18 & 31 \\
\hline
\end{tabular}

8

$9 \quad{ }^{a}$ Not sequenced. 